\title{
Bibliographical review of the main European unifloral honeys
}

\author{
Maria Gioia PIAZZA*, Livia PERSANo OdDO \\ Istituto Sperimentale per la Zoologia Agraria, Sezione di Apicoltura, Roma, Italy \\ (Received 20 February 2004; revised 11 May 2004; accepted 17 May 2004)
}

unifloral honey / Europe / bibliographic review / analytical properties

\section{INTRODUCTION}

The International Honey Commission of Apimondia (IHC) carried out a large work of characterization resulting in the descriptive sheets of 15 important European unifloral honey (Persano Oddo and Piro, 2004).

To compare the results obtained in IHC work with other European studies, an extensive bibliographic review was performed, collecting and tabulating the analytical data available in the literature related to the same honey types and analytical parameters reported in the descriptive sheets. Also some further unpublished data personally communicated by authors were included.

A summarising table was compiled for each of the 15 important European honey types. All data reported in the tables are generally the same as found in the original works. In some cases the original measure units were transformed to the official ones (Bogdanov et al., 1997), so that they can be compared to the data of the descriptive sheets (e.g. electrical conductivity in $\mathrm{mS} / \mathrm{cm}$, invertase in $\mathrm{U} / \mathrm{kg}$, etc.).

\footnotetext{
* Corresponding author: mg.piazza @ apicoltura.org
}

The complete references quoted in the review are listed in the reference list. The distribution of the studies per country is reported in Table I.

The results of the IHC study (Persano Oddo and Piro, 2004) are fairly consistent with those available in the literature. When some major differences are encountered, they are probably accounted for by the different analytical methods used.

The review provides a useful device for the knowledge and study of unifloral honeys.

\section{BIBLIOGRAPHIC DATA ON EUROPEAN UNIFLORAL HONEYS}

Legend. Numbers in bold = average values; the sign \pm precedes standard deviation values; numbers in round brackets $=\mathrm{min} / \mathrm{max}$ values; code in square brackets $=$ source of the data (see reference list). $\mathrm{PG}=$ pollen grains $; \mathrm{HDE}=$ Honeydew elements; $\mathrm{PE}=$ plant elements $(\mathrm{PG}+\mathrm{HDE})$. 
Table I. References per country.

\begin{tabular}{|l|l|}
\hline Belgium & {$[$ BRU 92] } \\
\hline Bulgaria & [DIN 03] [IVA 78] [IVA 97] [IVA 02] \\
\hline Croatia & [MUR 76] \\
\hline Czech Republic & [ČEL 01] \\
\hline Denmark & [RAV 75] \\
\hline France & [FAU 02] [GON 79] [GON 83] [GON 87] [ITA 75] [POU 92] \\
\hline Germany & [DUS 67] [MAU 64] [VDO 96] [VOR 64] \\
\hline Greece & [DRI 95] [KAR 97] [THR 95] [TSI 00] [MAN 01] \\
\hline Hungary & [FOL 94] [FOL 96] \\
\hline Italy & [PER 95A] [PER 95B] [PER 99] [PER 00] \\
\hline Poland & [KRA 91] \\
\hline Portugal & [RUS 97] [MEN 98] \\
\hline Romania & [BAC 65] \\
\hline Slovenia & [GOL 99] [GOL 03] \\
\hline Spain & $\begin{array}{l}\text { [CAB 97] [ESP 81] [JAT 95] [JUA 92] [MAT 92] [MAT 97] [MAT 98] [ORT 95] [PEZ 95A] [PEZ } \\
\text { 95B] [PEZ 90] [PUJ 94] [SAN 01] [SAU 82A] [SAU 82B] [SER 87] [SER 88A] [SER 88B] [SER 88C] } \\
\text { [SER 93] [SER 95] [SER 00] }\end{array}$ \\
\hline Switzerland & [BOG 88] [BOG 90] [BOG 97] \\
\hline Yugoslavia & [SHL 81] \\
\hline Europe & [BOG 99] [STA 74] \\
\hline
\end{tabular}

\section{BRASSICA}

\begin{tabular}{|c|c|}
\hline $\mathrm{PG} / 10 \mathrm{~g}$ honey $\cdot 10^{3}$ & $>100[$ STA 74] \\
\hline$\%$ Specific pollen & $\begin{array}{l}\text { very high [ITA 75]; (45-90) [BOG 90]; >65 [BRU 92]; >45 [PEZ 95A]; 65 } \pm 27 \\
\text { [BOG 97] }\end{array}$ \\
\hline Colour (mm Pfund) & 35 [ITA 75]; 29 $\pm 11(11 / 41)$ [BRU 92] \\
\hline $\begin{array}{l}\text { Electrical conductivity } \\
(\mathrm{mS} / \mathrm{cm})\end{array}$ & $\begin{array}{l}\mathbf{0 . 1 4} \text { [VOR 64]; (0.17/0.21) [DUS 67]; (0.1/0.25) [ITA 75]; (0.12/0.55) [RAV 75]; } \\
(0.16 / 0.58) \text { [BOG 90]; 0.19 (0.13/0.36) [KRA 91]; 0.21 } \pm 0.06(0.10 / 0.33) \text { [BRU 92]; } \\
\mathbf{0 . 1 7} \pm 0.50(0.10 / 0.26) \text { [POU 92]; 0.26 } \pm 0.03 \text { [PEZ 95A]; 0.18 } \pm 0.04(0.10 / 0.29) \\
{[\text { VDO 96]; 0.34 } \pm 0.16 \text { [BOG 97]; }(0.09 / 0.27) \text { [BOG 99] }}\end{array}$ \\
\hline $\mathrm{pH}$ & $\begin{array}{l}\text { 4.0 (3.8/4.3) [ITA 75]; (3.24/4.31) [RAV 75]; } 4.1 \text { (4.0/4.2) [KRA 91]; 4.1 } \pm 0.2(3.9 / \\
\text { 4.3) [BRU 92]; 4.0 } \pm 0.1(3.8 / 4.2)[\mathrm{POU} 92] ; \mathbf{3 . 6}[\mathrm{PEZ} \mathrm{95A];} \mathrm{3.9 \pm 0.54} \mathrm{[BOG} \mathrm{97]}\end{array}$ \\
\hline Free acidity $(\mathrm{meq} / \mathrm{kg})$ & 13.2 (9.3/21.2) [KRA 91]; 9.4 $\pm 3.4(5.0 / 15.0)[\mathrm{BRU} 92] ; \mathbf{2 2 . 0} \pm 6.0$ [PEZ 95A] \\
\hline Lactones (meq/kg) & 7.8 (4.1/14.2) [KRA 91]; 1.85 \pm 0.18 [PEZ 95A] \\
\hline Total acidity (meq/kg) & 21.0 (15.1/31.3) [KRA 91]; 14.9 $\pm 2.9(10.0 / 20.0)$ [POU 92]; 23.9 \pm 6.21 [PEZ 95A] \\
\hline Water $(\mathrm{g} / 100 \mathrm{~g})$ & $\begin{array}{l}(17.6 / 18.8) \text { [DUS 67]; (17.1/23.7) [RAV 75]; (13.8/19.1) [BOG 90]; 20.0 (17.8/ } \\
21.3) \text { [KRA 91]; 17.1 } \pm 0.9(15.6 / 19.9) \text { [BRU 92]; 17.6 } \pm 1.1 \text { (16.1/0.8) [POU 92]; } \\
\text { 18.7 } \pm 0.78 \text { [PEZ 95A]; 16.6 } \pm 0.75 \text { (14.7/18.8) [VDO 96] }\end{array}$ \\
\hline Diastase (DN) & $\begin{array}{l}>10 \text { [ITA 75]; 10.0 (5.0/17.9) [KRA 91]; 20.0 } \pm 6.9(11.0 / 26.0) \text { [POU 92]; 46.6 } \pm 4.9 \\
\text { [PEZ 95A] }\end{array}$ \\
\hline
\end{tabular}




\begin{tabular}{|c|c|}
\hline Invertase (U/kg) & 77.1 (50.7/39.7) [KRA 91]; 132.9 \pm 33.8 [VDO 96] \\
\hline Proline $(\mathrm{mg} / \mathrm{kg})$ & $233.5(163.4 / 389.2)$ [KRA 91] \\
\hline Fructose $(g / 100 \mathrm{~g})$ & $\begin{array}{l}\text { 44.6* (40.5/46.3) [ITA 75]; 39.7 [MUR 76]; (36.9/40.2) [GON 79]; 36.9(36.1/37.6) } \\
\text { [KRA 91]; 36.7 } \pm 3.00(31.7 / 41.8) \text { [BRU 92]; 36.7 } \pm 1.20 \text { (33.7/38.0) [POU 92]; } \\
\text { 35.3 } \pm 0.71 \text { [PEZ 95A]; 38.2 } \pm 1.64 \text { (31.1/42.0) [VDO 96]; 38.1 } \pm 1.50 \text { [BOG 97] }\end{array}$ \\
\hline Glucose $(\mathrm{g} / 100 \mathrm{~g})$ & $\begin{array}{l}\text { 48.2* (44.7/53.2) [ITA 75]; 35.1 [MUR 76]; (39.6/42.6) [GON 79]; 40.0 [GON 87]; } \\
\text { (32.2/37.1) [BOG 90]; 39.3 (38.6/39.7) [KRA 91]; 38.9 } \pm 2.7 \text { (34.9/42.6) [BRU 92]; } \\
\text { 39.7 } \pm 1.2 \text { (37.6/43.2) [POU 92]; 38.1 } \pm 3.7 \text { [PEZ 95A]; 40.2 } \pm 2.1 \text { (36.2/46.7) [VDO } \\
\text { 96]; 34.5 } \pm 1.6 \text { [BOG 97] }\end{array}$ \\
\hline Sucrose $(g / 100 \mathrm{~g})$ & $\begin{array}{l}\mathbf{0 . 5 4} *(0 / 1)[\text { ITA } 75] ;<\mathbf{1}[\mathrm{GON} 87] ;(0.1 / 0.2)[\mathrm{BOG} 90] ; \mathbf{0 . 4 9}(0.2 / 0.7)[\mathrm{KRA} 91] ; \\
\mathbf{0 . 5 0} \pm 0.20(0.0 / 0.9)[\mathrm{POU} 92] ; \mathbf{0 . 2 0} \pm 0.30(0.0 / 0.9)[\mathrm{BRU} 92] ; \mathbf{0 . 0 7} \pm 0.03[\mathrm{PEZ} \\
95 \mathrm{~A}] ; \mathbf{0 . 0 1}(0 / 0.3)[\text { VDO 96]; } \mathbf{0 . 1 2} \pm 0.07 \text { [BOG 97]; (0.0/1.0) [BOG 99] }\end{array}$ \\
\hline $\mathrm{F}+\mathrm{G}(\mathrm{g} / 100 \mathrm{~g})$ & 70.2** [MUR 76]; 76.1 (76.2/77.3) [KRA 91]; (68.2/83.9) [BOG 99] \\
\hline $\mathrm{F} / \mathrm{G}$ ratio & $\begin{array}{l}\mathbf{0 . 9 0}(0.76 / 1.0)[\text { ITA } 75] ; \mathbf{0 . 9 5} \pm 0.09(0.75 / 1.16)[B R U ~ 92] ; \mathbf{0 . 9 0} \pm 0.04(0.87 / 0.99) \\
{[\text { POU 92]; 0.93 } \pm 0.07 \text { [PEZ 95A]; } \mathbf{0 . 9 6} \pm 0.04(0.86 / 1.02) \text { [VDO 96]; 1.51 } \pm 0.09} \\
\text { [BOG 97] }\end{array}$ \\
\hline G/W ratio & $2.25(1.86 / 2.56)$ [ITA 75] \\
\hline
\end{tabular}

\section{CALLUNA}

\begin{tabular}{|c|c|}
\hline $\mathrm{PG} / 10 \mathrm{~g}$ honey $\cdot 10^{3}$ & (100 to 500) [SER 93] \\
\hline$\%$ Specific pollen & $17(10 / 33)$ [SER 93] \\
\hline $\begin{array}{l}\text { Electrical conductivity } \\
(\mathrm{mS} / \mathrm{cm})\end{array}$ & $(0.63 / 1.16)$ [RAV 75]; $\mathbf{0 . 5 0}(0.26 / 0.71)$ [KRA 91]; $\mathbf{0 . 8 1} \pm 0.16$ (0.59/1.22) [SER 93] \\
\hline $\mathrm{pH}$ & (3.95/5.00) [RAV 75]; 4.4 (3.98/4.98) [KRA 91]; 4.1 \pm 0.19 (3.7/4.3) [SER 93] \\
\hline Free acidity (meq/kg) & $22.7(11.5 / 45.2)$ [KRA 91]; 42.3 $\pm 6.2(29.0 / 52.2)$ [SER 93] \\
\hline Lactones (meq/kg) & 7.3 (4.7/12.1) [KRA 91]; 3.3 $\pm 2.2(0.48 / 7.95)$ [SER 93] \\
\hline Total acidity (meq/kg) & 30.0 (18.2/54.5) [KRA 91]; 45.5 $\pm 6.9(38.9 / 60.1)$ [SER 93] \\
\hline Water $(\mathrm{g} / 100 \mathrm{~g})$ & (19.2/24.0) [RAV 75]; 19.0 (16.4/21.6) [KRA 91]; 17.4 \pm 2.05 (14.4/19.8) [SER 93] \\
\hline Diastase (DN) & $24.4(8.3 / 38.5)$ [KRA 91]; 51.9 \pm 14.7 (26.1/81.1) [SER 93] \\
\hline Invertase $(\mathrm{U} / \mathrm{kg})$ & $120.5(41.9 / 191.7)$ [KRA 91] \\
\hline Proline (mg/kg) & 506.1 (289.4/862.3) [KRA 91] \\
\hline Fructose $(g / 100 g)$ & 39.5 (37.1/40.9) [KRA 91]; 43.3 $\pm 2.0(38.2 / 47.5)$ [SER 93] \\
\hline Glucose (g/100 g) & 31.9 (30.3/33.5) [KRA 91]; 31.0 $\pm 2.13(27 / 34.4)$ [SER 93] \\
\hline Sucrose $(\mathrm{g} / 100 \mathrm{~g})$ & $\mathbf{0 . 5 9}(0.33 / 0.86)[$ KRA 91$] ; \mathbf{0 . 1 6} \pm 0.08$ [SER 93] \\
\hline $\mathrm{F}+\mathrm{G}(\mathrm{g} / 100 \mathrm{~g})$ & 71.5 (67.4/73.9) [KRA 91] \\
\hline F/G ratio & $\mathbf{1 . 4 0} \pm 0.07(1.29 / 1.51)$ [SER 93] \\
\hline G/W ratio & 1.49 $\pm 0.23(1.16 / 1.90)[$ SER 93] \\
\hline
\end{tabular}




\section{CASTANEA}

\begin{tabular}{|c|c|}
\hline $\mathrm{PG} / 10 \mathrm{~g}$ honey $\cdot 10^{3}$ & $>100$ [PER 00] \\
\hline$\%$ Specific pollen & (95/99) [BOG 90]; >70 [JAT 95]; 98 \pm 1 [BOG 97]; >90 [PER 00] \\
\hline Colour (mm Pfund) & 70 $\pm 11(55 / 83)$ [BRU 92]; 92 \pm 9 (62/119) [PER 95A]; 89.1 \pm 16.9 [PER 00] \\
\hline $\begin{array}{l}\text { Electrical Conductivity } \\
(\mathrm{mS} / \mathrm{cm})\end{array}$ & $\begin{array}{l}(0.58 / 0.74) \text { [IVA 78]; }(0.67 / 1.92) \text { [BOG 90]; } \mathbf{0 . 9 6} \pm 0.23(0.68 / 1.24) \text { [BRU 92]; } \mathbf{1 . 2 4} \pm 0.09 \\
(1.11 / 1.42) \text { [PUJ 94]; }(0.88 / 2.15) \text { [JAT 95]; } \mathbf{1 . 4 1} \pm 0.24(1.01 / 2.09) \text { [PER 95A]; } \mathbf{1 . 5 4} \pm 0.32 \\
(1.11 / 2.06) \text { [THR 95]; 1.19 (0.85/1.50) [FOL 96]; 1.30 } \pm 0.34 \text { [BOG 97]; }(0.80 / 2.07) \\
\text { [BOG 99]; 1.48 } \pm 0.34(0.98 / 2.03) \text { [GOL 99]; 1.40 } \pm 0.24 \text { [PER 00] }\end{array}$ \\
\hline Specific rotation $[\alpha]_{D}^{20}$ & $(-2.3 /-3.5)$ [IVA 78]; -17.0 $\pm 3.5(-24.9 /-10.0)$ [PER 95A]; -16.4 \pm 3.4 [PER 00] \\
\hline $\mathrm{pH}$ & 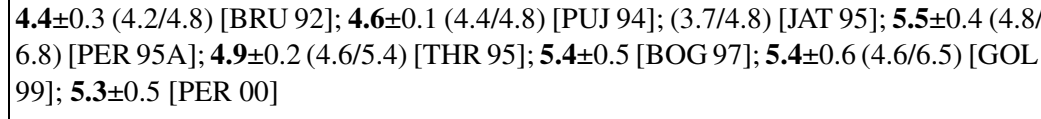 \\
\hline Free acidity $(\mathrm{meq} / \mathrm{kg})$ & $\begin{array}{l}\mathbf{2 6 . 3} \pm 4.0(23.0 / 32.0) \text { [BRU 92]; 48.2 } \pm 6.9 \text { (31.5/56.5) [PUJ 94]; (24.4/72.8) [JAT 95]; } \\
\mathbf{1 0 . 3}(9.3 / 11.3) \text { [FOL 96]; 17.4 } \pm 4.9(10.3 / 24.8) \text { [GOL 99]; 13.4 } \pm 3.3 \text { [PER 00] }\end{array}$ \\
\hline Lactones $(\mathrm{meq} / \mathrm{kg})$ & 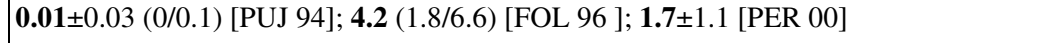 \\
\hline Total acidity $(\mathrm{meq} / \mathrm{kg})$ & $\begin{array}{l}\text { (8.5/13.0) [IVA 78]; 48.2 } \pm 6.9(31.5 / 56.5) \text { [PUJ 94]; 13.8 } \pm 3.8 \text { (5.7/24.0) [PER 95A]; } \\
\mathbf{1 4 . 5}(11.1 / 18.0) \text { [FOL 96]; 15.1 } \pm 4.0 \text { [PER 00] }\end{array}$ \\
\hline Water $(g / 100 \mathrm{~g})$ & $\begin{array}{l}(17.1 / 19.3)[\text { IVA 78]; (18.0/19.0) [SHL 81]; (15.6/18.7) [BOG 90]; 18.9 } \pm 2.2(16.8 / 22.0) \\
\text { [BRU 92]; 16.2 } \pm 0.6(15.6 / 17.8)[\text { PUJ 94]; }(16.6 / 19.1)[\text { JAT 95]; 17.4 } \pm 0.9(<19) \\
\text { [PER 95A]; 16.3 } \pm 0.71(14.9 / 17.4)[\text { THR 95]; 15.4 } \pm 0.7(14.6 / 16.9)[G O L ~ 99] ; 17.5 \pm 0.9 \\
\text { [PER 00] }\end{array}$ \\
\hline Diastase $(\mathrm{DN})$ & $\begin{array}{l}\text { (13.0/18.4) [IVA 78]; (18.9/33.2) [JAT 95]; 23.9 } \pm 5.0(10.6 / 42.9) \text { [PER 95A]; 32.2 } \pm 8.91 \\
(23.9 / 51.0) \text { [THR 95]; 17.4 } \pm 2.5 \text { (12.7/21.8) [GOL 99]; 24.5 } \pm 5.2 \text { [PER 00] }\end{array}$ \\
\hline Invertase $(\mathrm{U} / \mathrm{kg})$ & 158.7 $\pm 29.4(108.0 / 214.5)[$ PER 99, PER 00]; 188.0 $\pm 42.6(141.0 / 239.4)$ [SER 00] \\
\hline Proline $(\mathrm{mg} / \mathrm{kg})$ & $\begin{array}{l}\text { (71/526) [JAT 95]; 554 } \pm 139 \text { (432/734) [THR 95]; } 733 \text { (662/943) [FOL 96]; 590 } \pm 150 \\
\text { [PER 00]; (573/1292) [SAN 01] }\end{array}$ \\
\hline Fructose $(\mathrm{g} / 100 \mathrm{~g})$ & $\begin{array}{l}\text { (35.0/44.7) [SHL 81]; 37.9 } \pm 2.7 \text { (34.7/41.2) [BRU 92]; (35.1/43.5) [JAT 95]; 41.9 } \pm 2.1 \\
\text { (37.1/47.4) [PER 95A]; 41.9 } \pm 1.3 \text { [BOG 97]; 41.5 } \pm 2.4 \text { (37.6/46.0) [GOL 99]; 41.9 } \pm 2.0 \\
\text { [PER 00] }\end{array}$ \\
\hline Glucose $(g / 100 \mathrm{~g})$ & $\begin{array}{l}(25.2 / 30.8) \text { [SHL 81]; (25.6/30.5) [BOG 90]; 29.5 } \pm 0.8(28.8 / 30.6) \text { [BRU 92]; (21.0/30.0) } \\
\text { [JAT 95]; 26.4 } \pm 1.4(22.1 / 29.3) \text { [PER 95A]; 27.4 } \pm 1.13 \text { [BOG 97]; 29.4 } \pm 2.15(23.6 / 32.4) \\
\text { [GOL 99]; 26.4 } \pm 1.5 \text { [PER 00] }\end{array}$ \\
\hline Sucrose $(g / 100 \mathrm{~g})$ & $\begin{array}{l}(0 / 6.2) \text { [IVA 78]; (0.1/3.8) [BOG 90]; traces [BRU 92]; (0/0.20) [JAT 95]; 0.1 } \pm 0.1 \text { [PER } \\
95 \mathrm{~A}] ; \mathbf{0 . 5 0} \pm 0.65 \text { [BOG 97]; (0.0/1.3) [BOG 99]; 0.77 } \pm 0.23(0.47 / 1.17) \text { [GOL 99]; } \\
\mathbf{0 . 0 4} \pm 0.06 \text { [PER 00] }\end{array}$ \\
\hline $\mathrm{F}+\mathrm{G}(\mathrm{g} / 100 \mathrm{~g})$ & $\begin{array}{l}(64.3 / 74.3) \text { [JAT 95]; 68.2 } \pm 2.7 \text { (62.0/75.7) [PER 95A]; (62.0/81.4) [BOG 99]; 70.8 } \pm 3.94 \\
(61.9 / 78.4) \text { [GOL 99]; 68.3 } \pm 2.7 \text { [PER 00] }\end{array}$ \\
\hline $\mathrm{F} / \mathrm{G}$ ratio & $\begin{array}{l}\mathbf{1 . 2 8} \pm 0.06(1.20 / 1.35) \text { [BRU 92]; (1.20/1.90) [JAT 95]; 1.59 } \pm 0.11 \text { (1.34/1.95) [PER 95A, } \\
\text { PER 00]; 1.54 } \pm 0.09 \text { [BOG 97]; 1.42 } \pm 0.10(1.32 / 1.62)[\text { GOL 99] }\end{array}$ \\
\hline $\mathrm{G} / \mathrm{W}$ ratio & $(1.24 / 1.81)$ [JAT 95]; 1.51 $\pm 0.13(1.14 / 1.84)$ [PER 95A, PER 00] \\
\hline
\end{tabular}




\section{CITRUS}

\begin{tabular}{|c|c|}
\hline $\mathrm{PG} / 10 \mathrm{~g}$ honey $\cdot 10^{3}$ & $<20$ [SER 87, SER 95, PER 00] \\
\hline$\%$ Specific pollen & $>15$ [SER 87]; >10 [PER 00] \\
\hline Colour (mm Pfund) & $\begin{array}{l}\text { 17 (17/34) [SER 87]; 30 (11/55) [MAT 92]; 14 } \pm 5 \text { (11/35) [PER 95A]; 11.7 } \pm 5.2 \text { [SER 95]; } \\
\text { 14.3 } \pm 5.5 \text { [PER 00] }\end{array}$ \\
\hline $\begin{array}{l}\text { Electrical conductivity } \\
(\mathrm{mS} / \mathrm{cm})\end{array}$ & $\begin{array}{l}\mathbf{0 . 2 6} \pm 0.02[\text { ESP } 81] ; \mathbf{0 . 2 2} \pm 0.04(0.14 / 0.31)[\text { POU } 92] ; \mathbf{0 . 1 7} \pm 0.04(0.10 / 0.25)[\text { PER 95A]; } \\
\text { 0.19 } \pm 0.38(0.15 / 0.31)[\text { THR 95]; 0.23 (0.17/0.29) [CAB 97]; 0.28 } \pm 0.57(0.19 / 0.43) \\
{[\text { KAR 97]; 0.18 } \pm 0.03(0.12 / 0.26)[\text { MAT 98]; (0.10/0.35) [BOG 99]; 0.18 } \pm 0.04 \text { [PER 00] }}\end{array}$ \\
\hline Specific rotation $[\alpha]_{\mathrm{D}}^{20}$ & $-\mathbf{- 1 4 . 0} \pm 2.0(-17.7 /-9.3)[\mathrm{PER} 95 \mathrm{~A}] ; \mathbf{- 1 3 . 5} \pm 2.1[\mathrm{PER} 00]$ \\
\hline $\mathrm{pH}$ & $\begin{array}{l}\text { 3.8 } \pm 0.1 \text { [ESP 81]; 3.8 } \pm 0.1(3.5 / 4.1) \text { [POU 92]; 3.9 } \pm 0.1 \text { (3.7/4.2) [PER 95A, PER 00]; } \\
\text { 3.4 } \pm 0.05(3.3 / 3.6)[T H R ~ 95] ; \mathbf{3 . 4} \pm 0.24(3.0 / 3.8)[\text { KAR 97]; 4.0 } \pm 0.1 \text { (3.8/4.1) [MAT 98] }\end{array}$ \\
\hline Free acidity (meq/kg) & 24.7 \pm 1.8 [ESP 81]; 18.5 (12.9/24.2) [CAB 97]; 14.4 \pm 3.2 [PER 00] \\
\hline Lactones (meq/kg) & 2.0 \pm 0.2 [ESP 81]; $3.4(0.60 / 5.02)$ [CAB 97]; 3.0 \pm 2.3 [PER 00] \\
\hline Total acidity (meq/kg) & $\begin{array}{l}\text { 26.7 } \pm 1.7 \text { [ESP 81]; 22.0 } \pm 5.9(12.6 / 33.8) \text { [POU 92]; 17.0 } \pm 4.1(10.0 / 25.0) \text { [PER 95A]; } \\
\text { 22.1 (13.5/29.2) [CAB 97]; 17.4 } \pm 3.7 \text { [PER 00] }\end{array}$ \\
\hline Water $(g / 100 \mathrm{~g})$ & $\begin{array}{l}\text { 17.9 } \pm 0.7 \text { [ESP } 81] ; \mathbf{1 9 . 1} \pm 1.44(17.3 / 21.4)[\text { SER 87]; 17.3 } \pm 1.2(<19) \text { [PER 95A]; } \\
\text { 17.2 } \pm 0.88 \text { [SER 95]; 16.9 } \pm 0.66(16.0 / 18.5)[\text { THR 95]; 17.6 }(15.3 / 21.6) \text { [CAB 97]; } \\
\text { 17.8 } \pm 0.98(15.9 / 20.1) \text { [KAR 97]; 18.2 } \pm 0.93(16.4 / 19.6) \text { [MAT 98]; 18.2 } \pm 1.40(15.4 / \\
\text { 20.9) [POU 92]; 16.4 } \pm 0.90 \text { [PER 00] }\end{array}$ \\
\hline Diastase (DN) & $\begin{array}{l}\text { (13.1/21.5) [SER 88B]; 9.3 } \pm 2.8(3.4 / 16.3) \text { [PER 95A]; 17.6 } \pm 2.69 \text { [SER 95]; 11.7 } \pm 3.78(8.6 / \\
\text { 15.5) [THR 95]; 9.9 (1.8/21.1) [CAB 97]; 13.8 } \pm 5.35 \text { (6.1/40.6) [KAR 97]; 8.9 } \pm 2.6 \text { [PER 00] }\end{array}$ \\
\hline Invertase (U/kg) & $\begin{array}{l}\text { 22.8 } \pm 8.8(12.5 / 52.9)[\text { KAR 97]; 38.9 } \pm 16.9 \text { (7.3/69.8) [PER 99]; 39.7 } \pm 16.9 \text { [PER 00]; } \\
\mathbf{6 6 . 8} \pm 14.8(51.2 / 90.0) \text { [SER 00] }\end{array}$ \\
\hline Proline $(\mathrm{mg} / \mathrm{kg})$ & $\begin{array}{l}\mathbf{3 2 6} \pm 134(264 / 636) \text { [THR 95]; } 415(140 / 655) \text { [CAB 97]; 861 } \pm 88 \text { (752/975) [KAR 97]; } \\
\mathbf{2 3 0} \pm 90 \text { [PER 00] }\end{array}$ \\
\hline Fructose $(g / 100 g)$ & $\begin{array}{l}\text { 42.6* } \pm 1.5 \text { [ESP 81]; 35.4 } \pm 1.5(32.8 / 38.6) \text { [SER 87]; 37.7 } \pm 1.1 \text { (35.7/39.6) [POU 92]; } \\
\text { 38.8 } \pm 3.0(33.5 / 45.1)[\text { PER 95A]; 33.3 } \pm 1.2 \text { [SER 95]; 38.5 (35.2/44.5) [CAB 97]; } \\
\text { 36.7 } \pm 2.0(31.9 / 39.1) \text { [MAT 97, MAT 98]; 38.4 } \pm 2.6[\text { PER 00] }\end{array}$ \\
\hline Glucose $(g / 100 \mathrm{~g})$ & $\begin{array}{l}\text { 39.2 } * \pm 1.4 \text { [ESP } 81] ; \mathbf{2 9 . 7} \pm 1.48(27.4 / 32.8) \text { [SER 87]; 31.0 } \pm 1.0(29.0 / 33.9) \text { [POU 92]; } \\
\text { 32.2 } \pm 1.3(29.8 / 35.7) \text { [PER 95A]; 28 } \pm 0.97 \text { [SER 95]; 30.5 (27.0/33.8) [CAB 97]; } \\
\text { 30.2 } \pm 2.3(25.3 / 34.3) \text { [MAT 97, MAT 98]; 32.0 } \pm 1.6 \text { [PER 00] }\end{array}$ \\
\hline Sucrose $(g / 100 \mathrm{~g})$ & $\begin{array}{l}\mathbf{0 . 9} * \pm 0.1 \text { [ESP } 81] ; \mathbf{2 . 9 7} \pm 1.7(0.24 / 5.7) \text { [SER } 87] ; \mathbf{1 . 6 0} \pm 1.8(0.0 / 7.5)[\text { POU 92]; } \mathbf{0 . 9 0} \pm 1.1 \\
(0 / 4.5) \text { [PER 95A]; 13.4 } \pm 2.4 \text { [SER 95]; 4.45 } \pm 3.3(1.05 / 12.0) \text { [MAT 97, MAT 98]; } \\
\mathbf{1 . 0 0} \pm 1.2 \text { [PER 00] }\end{array}$ \\
\hline $\mathrm{F}+\mathrm{G}(\mathrm{g} / 100 \mathrm{~g})$ & 71.0 $\pm 3.6(63.7 / 77.9)$ [PER 95A]; 71.9 (67.1/76.1) [CAB 97]; 70.4 \pm 3.6 [PER 00] \\
\hline $\mathrm{F} / \mathrm{G}$ ratio & $\begin{array}{l}\mathbf{1 . 1 0} \pm 0.07[\mathrm{ESP} 81] ; \mathbf{1 . 2 1} \pm 0.09(1.16 / 1.28)[\mathrm{POU} 92] ; \mathbf{1 . 2 0} \pm 0.09(1.02 / 1.43)[\mathrm{PER} 95 \mathrm{~A}] \\
\mathbf{1 . 2 6}(1.12 / 1.55)[\mathrm{CAB} 97] ; \mathbf{1 . 2 1} \pm 0.05(1.12 / 1.30)[\mathrm{MAT} 97] ; \mathbf{1 . 2 0} \pm 0.08 \text { [PER 00] }\end{array}$ \\
\hline $\mathrm{G} / \mathrm{W}$ ratio & $\begin{array}{l}\mathbf{1 . 8 7} \pm 0.13(1.58 / 2.12)[\text { PER 95A]; } \mathbf{1 . 7 6}(1.30 / 2.04)[\text { CAB 97]; 1.66 } \pm 0.16(1.39 / 1.91) \\
{[\text { MAT 97, MAT 98]; 1.90 } \pm 0.16 \text { [PER 00] }}\end{array}$ \\
\hline
\end{tabular}




\section{EUCALYPTUS}

\begin{tabular}{|c|c|}
\hline $\mathrm{PG} / 10 \mathrm{~g}$ honey $\cdot 10^{3}$ & $>100$ [SER 88C, PER 00] \\
\hline$\%$ Specific pollen & $>74$ [SER $88 \mathrm{C}] ;>90$ [PER 00] \\
\hline Colour (mm Pfund) & 71 (51/83) [MAT 92]; 58 \pm 11 (41/71) [PER 95A]; 55 \pm 10 [PER 00] \\
\hline $\begin{array}{l}\text { Electrical } \\
\text { conductivity }(\mathrm{mS} / \mathrm{cm})\end{array}$ & $\begin{array}{l}\mathbf{0 . 5 9} \pm 0.1(0.43 / 0.79)[\text { SER } 88 C] ; \mathbf{0 . 5 0} \pm 0.08(0.36 / 0.71)[\text { PER 95A]; } \mathbf{0 . 4 6} \pm 0.09(0.34 / \\
0.66) \text { [MAT 98]; (0.19/1.33) [BOG 99]; 0.48 } \pm 0.06 \text { [PER 00] }\end{array}$ \\
\hline Specific rotation $[\alpha]_{\mathrm{D}}^{20}$ & $-\mathbf{1 4} \pm 2.1(-17.1 /-11.8)[$ PER 95A]; $\mathbf{- 1 3 . 4 \pm 2 . 3 ~ [ P E R ~ 0 0 ] ~}$ \\
\hline $\mathrm{pH}$ & 3.9 $\pm 0.1(3.7 / 4.1)$ [PER 95A, PER 00]; 4.1 \pm 0.18 (3.82/4.35) [MAT 98] \\
\hline Free acidity $(\mathrm{meq} / \mathrm{kg})$ & 21.0 $\pm 4.3(13.3 / 30.9)[$ SER 88C]; 19.5 \pm 5.1 [PER 00] \\
\hline Lactones $(\mathrm{meq} / \mathrm{kg})$ & 7.3 $\pm 1.3(4.38 / 9.62)$ [SER 88C]; 3.3 \pm 2.4 [PER 00] \\
\hline Total acidity $(\mathrm{meq} / \mathrm{kg})$ & 28.3 $\pm 5.22(18.41 / 39.71)[$ SER $88 C] ; \mathbf{2 4 . 1} \pm 5.0(14.7 / 32.2)[$ PER 95A]; 22.1 $\pm 4.6[$ PER 00] \\
\hline Water $(\mathrm{g} / 100 \mathrm{~g})$ & $\begin{array}{l}\mathbf{1 6 . 8} \pm 1.04(14.8 / 18.9)[\text { SER } 88 C] ; \mathbf{1 6 . 5} \pm 1.0(<18)[\text { PER 95A]; 15.7 } \pm 0.83(14.1 / 16.7) \\
{[\text { MAT 98]; 15.7 } \pm 0.90[\text { PER 00] }}\end{array}$ \\
\hline Diastase (DN) & 18.0 $\pm 5.6(9.5 / 28.6)[$ SER $88 \mathrm{C}] ; \mathbf{2 6 . 1} \pm 4.9(16.2 / 34.9)$ [PER 95A]; 26.0 \pm 4.1 [PER 00] \\
\hline Invertase $(\mathrm{U} / \mathrm{kg})$ & 154.2 $\pm 28.6(99.2 / 207.9)$ [PER 99, PER 00]; 77.9 $\pm 36.0(46.3 / 117.5)$ [SER 00] \\
\hline Proline $(\mathrm{mg} / \mathrm{kg})$ & $\mathbf{4 7 0} * \pm 115(313 / 626)[$ SAN 01]; 571** $\pm 150(340 / 842)[$ SAN 01] \\
\hline Fructose $(\mathrm{g} / 100 \mathrm{~g})$ & $\begin{array}{l}\text { 38.6 } \pm 3.1 \text { (33.4/43.7) [PER 95A]; 38.7 } \pm 0.6(37.0 / 39.2) \text { [MAT 97, MAT 98]; 39.1 } \pm 2.3 \\
{[\text { PER 00] }}\end{array}$ \\
\hline Glucose $(g / 100 \mathrm{~g})$ & $\begin{array}{l}\text { 32.9 } \pm 1.0(31.2 / 34.5)[\text { PER 95A]; 31.7 } \pm 1.4(28.5 / 33.9) \\
{[\text { PMER 00] }}\end{array}$ \\
\hline Sucrose $(\mathrm{g} / 100 \mathrm{~g})$ & $\begin{array}{l}\text { 1.3 } \pm 0.8(0.1 / 2.8)[\text { PER 95A]; 0.3 } \pm 0.28(0.07 / 0.94) \text { [MAT 97, MAT 98]; (0.1/2.8) [BOG } \\
99] ; \mathbf{1 . 2} \pm 0.8 \text { [PER 00] }\end{array}$ \\
\hline $\mathrm{F}+\mathrm{G}(\mathrm{g} / 100 \mathrm{~g})$ & 71.5 $\pm 3.3(66.3 / 77.0)$ [PER 95A]; (63.3/77.0) [BOG 99]; 72.7 \pm 2.9 [PER 00] \\
\hline F/G ratio & $\begin{array}{l}\mathbf{1 . 1 7} \pm 0.10(1.01 / 1.40)[\mathrm{PER} 95 \mathrm{~A}] ; \mathbf{1 . 2 2} \pm 0.05(1.15 / 1.37)[\mathrm{MAT} 97, \text { MAT 98]; 1.16 } \pm 0.06 \\
{[\mathrm{PER} 00]}\end{array}$ \\
\hline G/W ratio & $\begin{array}{l}\mathbf{2 . 0 4} \pm 0.14(1.80 / 2.32)[\text { PER 95A]; 2.02 } \pm 0.14(1.71 / 2.26)[\text { MAT 97, MAT 98]; 2.17 } \pm 0.17 \\
{[\text { PER 00] }}\end{array}$ \\
\hline \multicolumn{2}{|c|}{$\begin{array}{l}* \text { data from Galizia } \\
* * \text { data from Basque Country }\end{array}$} \\
\hline
\end{tabular}




\section{HELIANTHUS}

\begin{tabular}{|c|c|}
\hline $\mathrm{PG} / 10 \mathrm{~g}$ honey $\cdot 10^{3}$ & $<50$ [PER 95A]; <30 [PER 00] \\
\hline$\%$ Specific pollen & (10/95) [POU 92]; >15 [PER 00] \\
\hline Colour (mm Pfund) & (35/75) [GON 87]; 67.5 (51/83) [MAT 92]; 61 \pm 6 (51/71) [PER 95A, PER 00] \\
\hline $\begin{array}{l}\text { Electrical conductivity } \\
(\mathrm{mS} / \mathrm{cm})\end{array}$ & $\begin{array}{l}\mathbf{0 . 3 1}[\text { IVA } 78] ; \mathbf{0 . 3 1}(0.23 / 0.45)[\mathrm{POU} 92] ; \mathbf{0 . 2 4} \pm 0.08(0.12 / 0.40)[\mathrm{ORT} 95] ; \mathbf{0 . 3 5} \pm 0.04 \\
(0.28 / 0.43)[\mathrm{PER} 95 \mathrm{~A}] ; \mathbf{0 . 4 3} \pm 0.12(0.26 / 0.57)[\mathrm{THR} 95] ; \mathbf{0 . 2 4}(0.20 / 0.28) \text { [FOL } 96] ; \\
\mathbf{0 . 4 1} \pm 0.55(0.29 / 0.55)[\mathrm{KAR} 97] ; \mathbf{0 . 3 7} \pm 0.06(0.28 / 0.46)[\mathrm{MAT} 98] ;(0.20 / 0.60)[\mathrm{BOG} \\
99] ; \mathbf{0 . 3 4} \pm 0.04[\mathrm{PER} 00] ; \mathbf{0 . 3 0}(0.23 / 0.49)[\mathrm{IVA} 02]\end{array}$ \\
\hline Specific rotation $[\alpha]_{\mathrm{D}}^{20}$ & $\mathbf{- 1 8} \pm 1.2(-19.8 /-15.4)[\mathrm{PER} 95 \mathrm{~A}] ; \mathbf{- 1 7 . 6} \pm 1.4$ [PER 00] \\
\hline $\mathrm{pH}$ & $\begin{array}{l}\text { 3.9 (3.6/4.1) [POU 92]; 3.8 } \pm 0.1 \text { (3.6/4.1) [PER 95A, PER 00]; 3.8 } \pm 0.12(3.6 / 4.0) \text { [THR } \\
95] ; \mathbf{3 . 4} \pm 0.33(2.9 / 4.0) \text { [KAR 97]; 3.9 } \pm 0.14 \text { (3.6/4.2) [MAT 98]; 3.8 (3.4/3.9) [IVA 02] }\end{array}$ \\
\hline Free acidity $(\mathrm{meq} / \mathrm{kg})$ & 26.1 $\pm 6.3[$ PER 00] \\
\hline Lactones (meq/kg) & 3.7 $\pm 3.0[$ PER 00] \\
\hline Total acidity $(\mathrm{meq} / \mathrm{kg})$ & $(16.0 / 48.0)$ [IVA 78]; 26.2 $\pm 5.4(16.0 / 35.2)$ [PER 95A]; 29.9 \pm 6.5 [PER 00] \\
\hline Water $(g / 100 \mathrm{~g})$ & $\begin{array}{l}(15.6 / 21.0) \text { [IVA 78]; 16.8 } \pm 0.7(<18)[\text { PER 95A]; 17.4 } \pm 0.84(16.5 / 19.5) \text { [THR 95]; } \\
\mathbf{1 8 . 4} \pm 1.31(15.0 / 20.6) \text { [KAR 97]; 17.0 } \pm 1.0(14.5 / 18.2) \text { [MAT 98]; 16.9 } \pm 0.8 \text { [PER 00]; } \\
\mathbf{1 8 . 2}(18.0 / 18.8) \text { [IVA 02] }\end{array}$ \\
\hline Diastase (DN) & $\begin{array}{l}(8.3 / 38.5) \text { [BAC 65]; (8.0/20.4) [IVA 78]; 15.4 } \pm 3.0(8.7 / 20.3) \text { [PER 95A]; 15.9 } \pm 3.17 \\
(9.3 / 23.0) \text { [THR 95]; 25.3 } \pm 6.2(14.4 / 44.2)[\text { KAR 97]; 17.7 } \pm 3.1[\text { PER 00] }\end{array}$ \\
\hline Invertase $(\mathrm{U} / \mathrm{kg})$ & 95.5 $\pm 17.6(66.1 / 119.7)$ [PER 99]; 94.8 \pm 16.9 [PER 00]; 160.1 (144.7/213.7) [IVA 02] \\
\hline Proline $(\mathrm{mg} / \mathrm{kg})$ & $\begin{array}{l}\mathbf{6 6 5} \pm 352(298 / 1199)[\text { THR 95]; 419(374/464) [FOL 96]; 493 } \pm 111 \text { (296/667) [KAR 97]; } \\
\mathbf{7 2 0} \pm 110 \text { [PER 00] }\end{array}$ \\
\hline Fructose $(g / 100 g)$ & $\begin{array}{l}(34.8 / 40.3)[\text { BAC 65]; 41.2 [MUR 76]; 38.5 (36.0/41.0) [POU 92]; 38.1 } \pm 2.2(35.1 / 41.6) \\
{[\text { ORT 95]; 39.2 } \pm 3.3(32.6 / 44.7)[\text { PER 95A]; 39.4 } \pm 0.7 \text { (38.3/40.6) [MAT 97, MAT 98]; }} \\
\text { 38.7 } \pm 3.0[\text { PER 00] }\end{array}$ \\
\hline Glucose (g/100 g) & $\begin{array}{l}\text { (34.7/42.3) [BAC 65]; 31.1 [MUR 76]; (37/39) [GON 87]; 37.5 (35.0/42.0) [POU 92]; } \\
\mathbf{3 1 . 2} \pm 1.9(28.1 / 34.2) \text { [ORT 95]; 37.6 } \pm 1.5(35.3 / 40.8) \text { [PER 95A]; 35.4 } \pm 1.4(32.9 / 37.8) \\
\text { [MAT 97, MAT 98]; 37.0 } \pm 1.5 \text { [PER 00]; (35.2/41.3) [MAN 01] }\end{array}$ \\
\hline Sucrose $(g / 100 \mathrm{~g})$ & $\begin{array}{l}\text { (1.3/3.6) [BAC 65]; (0.0/6.7) [IVA 78]; 0.20 (0.5 max) [POU 92]; 2.1 } \pm 2.1(0.0 / 7.0) \text { [ORT } \\
\text { 95]; } \mathbf{0 . 5} \pm 0.5 \text { (tr./1.8) [PER 95A]; } \mathbf{0 . 0 7 3} \pm 0.037(0.032 / 0.15) \text { [MAT 97, MAT 98]; (0.0/ } \\
\text { 1.8) [BOG 99]; 0.4 } \pm 0.5 \text { [PER 00] }\end{array}$ \\
\hline $\mathrm{F}+\mathrm{G}(\mathrm{g} / 100 \mathrm{~g})$ & $\begin{array}{l}(69.4 * / 77.8) \text { [IVA 78]; 76.8 } \pm 3.7 \text { (70.9/84.8) [PER 95A]; (68.7/84.8) [BOG 99]; } \\
\text { 75.8 } \pm 3.2 \text { [PER 00] }\end{array}$ \\
\hline F/G ratio & $\begin{array}{l}\mathbf{1 . 0 0}[\mathrm{GON} 87] ; \mathbf{1 . 0 5}(<1.10) \text { [POU 92]; 1.04 } \pm 0.10(0.84 / 1.16)[\mathrm{PER} 95 \mathrm{~A}] ; \mathbf{1 . 1 1} \pm 0.04 \\
(1.06 / 1.20) \text { [MAT 97, MAT 98]; 1.05 } \pm 0.10[\mathrm{PER} 00] ;(1.01 / 1.14)[\mathrm{MAN} 01]\end{array}$ \\
\hline $\mathrm{G} / \mathrm{W}$ ratio & $\begin{array}{l}\text { 2.24 } \pm 0.12(2.01 / 2.54)[\text { PER 95A]; 2.10 } \pm 0.18(1.83 / 2.50)[\text { MAT 97, MAT 98]; 2.19 } \pm 0.15 \\
{[\text { PER 00] }}\end{array}$ \\
\hline
\end{tabular}


LAVANDULA (L. vera, L. latifolia, L. angustifolia $\times$ latifolia)

\begin{tabular}{|c|c|}
\hline $\mathrm{PG} / 10 \mathrm{~g}$ honey $\cdot 10^{3}$ & $<20$ [SER 87] \\
\hline$\%$ Specific pollen & $>13$ [SER 87]; (2/10) [FAU 02] \\
\hline Colour (mm Pfund) & $\begin{array}{l}<5.5 \text { [ITA 75]; } 35 \text { [SAU 82B]; 34 (17/34) [SER 87, SER 88A]; 70 (46/99) [MAT 92]; 24 } \pm 6 \\
(11 / 45) \text { [POU 92] }\end{array}$ \\
\hline $\begin{array}{l}\text { Electrical } \\
\text { conductivity }(\mathrm{mS} / \mathrm{cm})\end{array}$ & $\begin{array}{l}\mathbf{0 . 2 5}(<0.4)[\text { ITA 75]; }(0.25 / 0.40)[\text { SAU } 82 B] ; \mathbf{0 . 4 2} \pm 0.17(0.19 / 0.97)[\text { SER } 87, \text { SER } 88 \mathrm{~A}] \\
\mathbf{0 . 2 3} \pm 0.04(0.15 / 0.37)[\mathrm{POU} 92] ;(0.12 / 0.60)[\text { BOG } 99]\end{array}$ \\
\hline $\mathrm{pH}$ & 3.6 (3.3/4.0) [ITA 75, SAU 82B]; 3.5 \pm 0.20 (3.2/3.9) [POU 92]; (3.3/3.9) [FAU 02] \\
\hline Free acidity $(\mathrm{meq} / \mathrm{kg})$ & 19.0*(11.6/26.2) [ITA 75, SAU 82B]; (10/20) [FAU 02] \\
\hline Lactones (meq/kg) & $15.3 *(10.0 / 21.4)[$ [TA 75, SAU 82B] \\
\hline Total acidity (meq/kg) & 34.2* (26.0/40.6) [ITA 75, SAU 82B]; 32.0 $\pm 4.2(22.0 / 42.0)$ [POU 92] \\
\hline Water $(g / 100 \mathrm{~g})$ & $\begin{array}{l}<17.5 \text { [ITA 75, SAU 82B]; 16.0 } \pm 0.43(15.1 / 17.0) \text { [SER 87, SER 88A]; 16.1 } \pm 1.00(14.9 / \\
\text { 18.4) [POU 92]; (15.3/17.8) [FAU 02] }\end{array}$ \\
\hline Diastase (DN) & $\begin{array}{l}>8 \text { [ITA 75, SAU 82B]; 45.4 } \pm 10.5(20.0 / 63.2) \text { [SER 87, SER 88A]; 22.0 } \pm 6.4(14.0 / 37.0) \\
\text { [POU 92]; (8/22.3) [FAU 02] }\end{array}$ \\
\hline Fructose $(g / 100 \mathrm{~g})$ & $\begin{array}{l}\text { 41.9**(39.2/45.4) [ITA 75, SAU 82B]; 37.8 } \pm 1.07 \text { (35.5/39.8) [SER 87, SER 88A]; } \\
\text { 35.0 } \pm 1.20(33.0 / 39.0) \text { [POU 92]; (33/40) [FAU 02] }\end{array}$ \\
\hline Glucose $(g / 100 \mathrm{~g})$ & $\begin{array}{l}\text { 38.7**(36.9/42.2) [ITA 75, SAU 82B]; 32.1 } \pm 1.44 \text { (27.8/34.3) [SER 87, SER 88A]; } \\
\text { 30.0 } \pm 1.30(28.0 / 34.0) \text { [POU 92]; (28/33) [FAU 02] }\end{array}$ \\
\hline Sucrose $(g / 100 \mathrm{~g})$ & $\begin{array}{l}\text { 7.22**(2.0/11.6) [ITA 75, SAU 82B]; 0.38 } \pm 0.44(0.025 / 2.02) \text { [SER 87, SER 88A]; } \\
\mathbf{7 . 5 0} \pm 2.10(2.0 / 12.0) \text { [POU 92]; (0.0/15.0) [BOG 99]; (1/13) [FAU 02] }\end{array}$ \\
\hline $\mathrm{F}+\mathrm{G}(\mathrm{g} / 100 \mathrm{~g})$ & $(60.1 / 73.2)$ [BOG 99] \\
\hline $\mathrm{F} / \mathrm{G}$ ratio & $\mathbf{1 . 0 8}(1.04 / 1.14)[$ ITA 75, SAU 82B]; 1.18 $\pm 0.04(1.13 / 1.23)$ [POU 92]; (1.1/1.3) [FAU 02] \\
\hline $\mathrm{G} / \mathrm{W}$ ratio & $2.00(1.80 / 2.20)[$ ITA 75] \\
\hline \multicolumn{2}{|l|}{$\begin{array}{l}* \mathrm{pH} \text { at equivalent point } \\
* * \% \text { of dry matter }\end{array}$} \\
\hline
\end{tabular}

\section{RHODODENDRON}

\begin{tabular}{|c|c|}
\hline $\mathrm{PG} / 10 \mathrm{~g}$ honey $\cdot 10^{3}$ & $<20$ [PER 00] \\
\hline$\%$ Specific pollen & (50/70) [BOG 90]; 53 \pm 13 [BOG 97]; >25 [PER 00] \\
\hline Colour (mm Pfund) & 13 $\pm 5(11 / 27)[$ PER 95A]; 13.1 \pm 4.8 [PER 00] \\
\hline $\begin{array}{l}\text { Electrical conductivity } \\
(\mathrm{mS} / \mathrm{cm})\end{array}$ & $\begin{array}{l}(0.22 / 0.35) \text { [BOG 90]; } \mathbf{0 . 2 2} \pm 0.05(0.15 / 0.33) \text { [PER 95A, PER 00]; } \mathbf{0 . 2 6} \pm 0.05 \\
\text { [BOG 97]; (0.15/0.45) [BOG 99] }\end{array}$ \\
\hline Specific rotation $[\alpha]_{D}^{20}$ & $-\mathbf{6} \pm 2.4(-10.7 /-2.3)[$ PER 95A]; $-\mathbf{6 . 1} \pm 2.5$ [PER 00] \\
\hline $\mathrm{pH}$ & 3.9 $\pm 0.1(3.8 / 4.2)$ [PER 95A, PER 00]; 3.9 \pm 1.7 [BOG 97] \\
\hline Free acidity $(\mathrm{meq} / \mathrm{kg})$ & 13.7 $\pm 3.4[$ PER 00] \\
\hline
\end{tabular}




\begin{tabular}{|c|c|}
\hline Lactones (meq/kg) & $1.3 \pm 1.3[$ PER 00] \\
\hline Total acidity (meq/kg) & 13.6 $\pm 3.0(7.4 / 18.9)[$ PER 95A]; 15 \pm 3.5 [PER 00] \\
\hline Water $(\mathrm{g} / 100 \mathrm{~g})$ & $(15.6 / 16.6)$ [BOG 90]; 16.6 $\pm 0.6(<18)$ [PER 95A]; 16.8 \pm 0.9 [PER 00] \\
\hline Diastase (DN) & 11.8 $\pm 2.4(7.1 / 16.7)[\mathrm{PER} 95 \mathrm{~A}] ; \mathbf{1 2 . 2} \pm 2.5$ [PER 00] \\
\hline Invertase (U/kg) & 81.5 $\pm 19.8(53.6 / 130.0)$ [PER 99]; 82.3 \pm 19.8 [PER 00] \\
\hline Proline (mg/kg) & $\mathbf{2 5 0} \pm 30[$ PER 00] \\
\hline Fructose $(\mathrm{g} / 100 \mathrm{~g})$ & 40.2 $\pm 2.2(36.5 / 45.5)[$ PER 95A]; 36.2 \pm 1.7 [BOG 97]; 38.0 \pm 2.6 [PER 00] \\
\hline Glucose $(\mathrm{g} / 100 \mathrm{~g})$ & $\begin{array}{l}(23.3 / 27.8) \text { [BOG 90]; 30.6 } \pm 2.4(27.2 / 34.8) \text { [PER 95A]; 25.5 } \pm 1.9 \text { [BOG 97]; } \\
\text { 30.2 } \pm 1.5 \text { [PER 00] }\end{array}$ \\
\hline Sucrose $(\mathrm{g} / 100 \mathrm{~g})$ & $\begin{array}{l}(0.9 / 4.3) \text { [BOG 90]; 0.5 } \pm 0.5(0 / 1.5) \text { [PER 95A]; 3.0 } \pm 1.3 \text { [BOG 97]; (0.9/4.3) } \\
\text { [BOG 99]; 0.4 } \pm 0.4[\text { PER 00] }\end{array}$ \\
\hline $\mathrm{F}+\mathrm{G}(\mathrm{g} / 100 \mathrm{~g})$ & 70.8 $\pm 4.0(63.7 / 79.4)$ [PER 95A]; (60.8/79.4) [BOG 99]; 68.1 \pm 3.3 [PER 00] \\
\hline $\mathrm{F} / \mathrm{G}$ ratio & $\mathbf{1 . 3 1} \pm 0.09(1.06 / 1.48)[\mathrm{PER} 95 \mathrm{~A}] ; \mathbf{1 . 4 2} \pm 0.14$ [BOG 97]; 1.26 \pm 0.09 [PER 00] \\
\hline G/W ratio & $\mathbf{1 . 8 3} \pm 0.14(1.62 / 2.12)[$ PER $95 \mathrm{~A}] ; \mathbf{1 . 8} \pm 0.1$ [PER 00] \\
\hline
\end{tabular}

\section{ROBINIA}

\begin{tabular}{|c|c|}
\hline $\mathrm{PG} / 10 \mathrm{~g}$ honey $\cdot 10^{3}$ & $<20$ [PER 00] \\
\hline$\%$ Specific pollen & low, very low [ITA 75]; (10/25) [BOG 90]; 17 [POU 92]; 20 \pm 5 [BOG 97]; >15 [PER 00] \\
\hline Colour (mm Pfund) & $<30$ [ITA 75]; 11 $\pm 0(11 / 11)$ [BRU 92]; 15 $\pm 6(11 / 27)$ [PER 95A]; 14.5 \pm 5.9 [PER 00] \\
\hline $\begin{array}{l}\text { Electrical } \\
\text { conductivity }(\mathrm{mS} / \mathrm{cm})\end{array}$ & 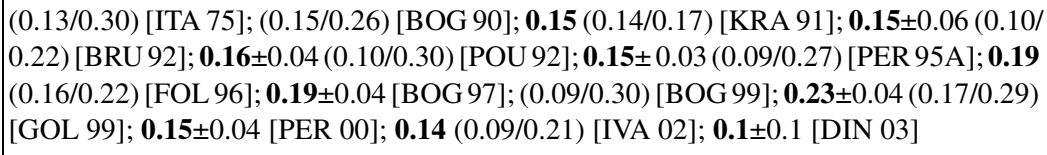 \\
\hline Specific rotation $[\alpha]_{D}^{20}$ & $\begin{array}{l}\mathbf{- 1 4 . 2}(-5.9 /-20.5)[\text { IVA 78]; } \mathbf{- 1 7 . 0} \pm 2.7(-23.4 /-10.9)[\text { PER 95A]; } \mathbf{- 1 6 . 9} \pm 2.67 \text { [PER 00]; } \\
\mathbf{- 1 5 . 1}(-6.1 /-19.5) \text { [IVA 02]; } \mathbf{- 1 7 . 0 \pm 1 . 2 ~ [ D I N ~ 0 3 ] ~}\end{array}$ \\
\hline $\mathrm{pH}$ & $\begin{array}{l}\text { 3.9 (3.7/4.1) [ITA 75]; 3.9 (3.8/4.0) [KRA 91]; 4.0 } \pm 0.2(3.8 / 4.2) \text { [BRU 92]; 3.9 } \pm 0.1(3.6 / \\
4.2)[\text { POU 92]; 4.0 } \pm 0.1(3.7 / 4.3)[\text { PER 95A]; 3.8 } \pm 0.25 \text { [BOG 97]; 3.7 } \pm 0.13(3.5 / 4.0) \\
\text { [GOL 99]; 3.9 } \pm 0.17 \text { [PER 00] }\end{array}$ \\
\hline $\begin{array}{l}\text { Free acidity } \\
(\mathrm{meq} / \mathrm{kg})\end{array}$ & $\begin{array}{l}\text { 8.0* (5.7/11.9) [ITA 75]; 13.8 } \pm 0.7(9.0 / 26.0) \text { [IVA 78]; 15.2 (10.6/18.2) [KRA 91]; 10.0 } \\
\text { [BRU 92]; 5.0 (3.9/5.9) [FOL 96]; 24.4 } \pm 2.71(20.1 / 31.0) \text { [GOL 99]; 10.9 } \pm 2.57 \text { [PER 00]; } \\
\mathbf{1 3 . 0} \pm 0.07(8.5 / 17.8) \text { [IVA 02] }\end{array}$ \\
\hline Lactones $(\mathrm{meq} / \mathrm{kg})$ & $\begin{array}{l}\text { 5.5* (2.2/9.4) [ITA 75]; } 4.9(4.2 / 6.0) \text { [KRA 91]; } 3.4(1.53 / 4.58) \text { [FOL 96]; 2.4 } \pm 1.77 \\
\text { [PER 00] }\end{array}$ \\
\hline $\begin{array}{l}\text { Total acidity } \\
(\mathrm{meq} / \mathrm{kg})\end{array}$ & $\begin{array}{l}\text { 13.7* }(8.9 / 20.4)[\text { ITA 75]; 20.0 }(16.3 / 22.7) \text { [KRA 91]; 14.3 } \pm 4.5 \text { (6.3/27.6) [POU 92]; } \\
\text { 12.4 } \pm 2.6(7.8 / 19.0)[\text { PER 95A]; 8.4 (5.4/10.0) [FOL 96]; 13.3 } \pm 3.4 \text { [PER 00] }\end{array}$ \\
\hline
\end{tabular}




\begin{tabular}{|c|c|}
\hline Water $(\mathrm{g} / 100 \mathrm{~g})$ & $\begin{array}{l}<\mathbf{1 8} \text { [ITA 75]; 18.2 } \pm 0.2(15.2 / 20.4) \text { [IVA 78]; }(13.5 / 19.1) \text { [BOG 90]; } 17.0(16.0 / 18.5) \\
{[\text { KRA 91]; 18.0 } \pm 0.3(17.7 / 18.3)[\text { BRU 92]; 17.6 } \pm 0.8(16.1 / 19.8) \text { [POU 92]; 16.4 } \pm 0.9} \\
(<18) \text { [PER 95A]; 16.5 } \pm 1(14.2 / 19.9) \text { [GOL 99]; 16.6 } \pm 0.9 \text { [PER 00]; 17.5 }(15.2 / 19.4) \\
{[\text { IVA 02]; 16.7 } \pm 0.9 \text { [DIN 03] }}\end{array}$ \\
\hline Diastase (DN) & $\begin{array}{l}>10(10 / 20)[\text { ITA 75]; 8.7 } \pm 0.4(5.2 / 14.8) \text { [IVA 78]; 11.5 (8.3/13.9) [KRA 91]; 8.6 } \pm 2.9(3.1 / \\
\text { 15.5) [PER 95A]; 9.3 } \pm 1.94(6.6 / 12.8) \text { [GOL 99]; 8.7 } \pm 2.7 \text { [PER 00]; 13.6 } \pm 0.8(8.0 / 21.0) \\
\text { [IVA 02] }\end{array}$ \\
\hline Invertase (U/kg) & $\begin{array}{l}\text { 51.4 (36.7/79.3) [KRA 91]; 26.4 } \pm 16.2(2.9 / 56.6)[\text { PER 99]; 27.2 } \pm 17.6 \text { [PER 00]; 29.4 } \pm 4.4 \\
(27.2 / 34.5) \text { [SER 00]; 27.9 } \pm 17.6 \text { [DIN 03] }\end{array}$ \\
\hline Proline (mg/kg) & $\begin{array}{l}\mathbf{2 2 9}(182.3 / 275.4) \text { [KRA 91]; } 199(189 / 283) \text { [FOL 96]; 210 } \pm 50 \text { [PER 00]; 213.4 } \pm 172.3 \\
\text { [DIN 03] }\end{array}$ \\
\hline Fructose $(g / 100 \mathrm{~g})$ & $\begin{array}{l}\text { 49.2**(44.7/53.7) [ITA 75]; 44.1 (40.7/48.1) [KRA 91]; 42.1 } \pm 2.2(40.0 / 44.3) \text { [BRU 92]; } \\
\text { 41.4 } \pm 1.3(39.0 / 44.0) \text { [POU 92]; 43.1 } \pm 2.9 \text { (36.9/48.5) [PER 95A]; 49.6 (47.7/51.2) [FOL } \\
\text { 96]; 41.6 } \pm 0.9 \text { [BOG 97]; (39.6/41.4) [IVA 97]; 43.7 } \pm 1.64 \text { (39.0/47.2) [GOL 99]; } \\
\text { 43.5 } \pm 2.37 \text { [PER 00] }\end{array}$ \\
\hline Glucose $(\mathrm{g} / 100 \mathrm{~g})$ & $\begin{array}{l}\text { 34.3**(29.2/38.0) [ITA 75]; (24.6/25.6) [BOG 90]; 28.4 (25.8/30.8) [KRA 91]; 29.2 } \pm 0.5 \\
(28.7 / 29.7) \text { [BRU 92]; 26.6 } \pm 1.3(22.6 / 29.2) \text { [POU 92]; 25.9 } \pm 1.5(21.0 / 28.8) \text { [PER 95A]; } \\
\text { 30.4 (28.7/32.9) [FOL 96]; 24.7 } \pm 0.6 \text { [BOG 97]; (23.3/23.7) [IVA 97]; 29.2 } \pm 1.1 \text { (26.9/ } \\
\text { 31.6) [GOL 99]; 26.1 } \pm 1.27 \text { [PER 00] }\end{array}$ \\
\hline Sucrose $(g / 100 \mathrm{~g})$ & $\begin{array}{l}\text { 2.5**(0.5/9.1) [ITA 75]; (2.0/4.8) [BOG 90]; 2.3(1.05/3.56) [KRA 91]; 0.4 } \pm 0.5(0.0 / 0.1) \\
\text { [BRU 92]; 1.8 } \pm 1.7(0.1 / 9.1) \text { [POU 92]; 2.5 } \pm 2.1(0.1 / 10.1) \text { [PER 95A]; 1.1 (0.1/3.8) [FOL } \\
\text { 96]; 3.2 } \pm 0.9 \text { [BOG 97]; (1.1/1.6) [IVA 97]; (0.0/10.0) [BOG 99]; 2.3 } \pm 1.63(0.92 / 6.40) \\
\text { [GOL 99]; 2.3 } \pm 1.9 \text { [PER 00] }\end{array}$ \\
\hline $\mathrm{F}+\mathrm{G}(\mathrm{g} / 100 \mathrm{~g})$ & $\begin{array}{l}\text { 72.6 (66.5/77.3) [KRA 91]; 69.0 } \pm 3.7 \text { (59.0/75.4) [PER 95A]; 79.9 (77.3/83.1) [FOL 96]; } \\
(64.3 / 64.8) \text { [IVA 97]; (60.6/83.8) [BOG 99]; 72.9 } \pm 2.2 \text { (67.5/78.8) [GOL 99]; 69.6 } \pm 3.0 \\
\text { [PER 00] }\end{array}$ \\
\hline $\mathrm{F} / \mathrm{G}$ ratio & $\begin{array}{l}\mathbf{1 . 4 3}(1.32 / 1.56)[\text { ITA } 75] ; \mathbf{1 . 4 5} \pm 0.10(1.35 / 1.54)[\mathrm{BRU} 92] ; \mathbf{1 . 5 5} \pm 0.09(1.38 / 1.79) \text { [POU } \\
92] ; \mathbf{1 . 6 7} \pm 0.11(1.38 / 1.89) \text { [PER 95A]; } \mathbf{1 . 6 3}(1.56 / 1.68) \text { [FOL 96]; } \mathbf{1 . 7 0} \pm 0.05 \text { [BOG 97]; } \\
(1.67 / 1.77) \text { [IVA 97]; } \mathbf{1 . 5 0} \pm 0.07(1.35 / 1.65) \text { [GOL 99]; 1.67 } \pm 0.10 \text { [PER 00] }\end{array}$ \\
\hline $\mathrm{G} / \mathrm{W}$ ratio & $\mathbf{1 . 6 3}(1.48 / 1.80)[$ ITA 75]; $\mathbf{1 . 5 5} \pm 0.11$ (1.24/1.82) [PER 95A]; $\mathbf{1 . 5 7} \pm 0.10$ [PER 00] \\
\hline \multicolumn{2}{|c|}{$\begin{array}{l}* \mathrm{pH} \text { at equivalent point } \\
* * \% \text { of dry matter }\end{array}$} \\
\hline
\end{tabular}

\section{ROSMARINUS}

\begin{tabular}{|c|c|}
\hline $\mathrm{PG} / 10 \mathrm{~g}$ honey $\cdot 10^{3}$ & $<20$ [PER 00] \\
\hline$\%$ Specific pollen & $>10$ [ITA 75, GON 83, PER 00] \\
\hline Colour (mm Pfund) & $<35$ [ITA 75]; 23 (11/51) [MAT 92]; $\mathbf{1 8 . 3} \pm 9.0(11 / 35)$ [PER 95B] 16.0 \pm 6.3 [PER 00] \\
\hline $\begin{array}{l}\text { Electrical conductivity } \\
(\mathrm{mS} / \mathrm{cm})\end{array}$ & $\begin{array}{l}<0.25[\text { ITA } 75] ;<0.25[\text { GON } 83] ; \mathbf{0 . 2 2} \pm 0.07(0.13 / 0.32)[\text { SER } 87] ; \mathbf{0 . 1 6} \pm 0.01 \text { [PEZ 90]; } \\
\mathbf{0 . 1 4} \pm 0.04(0.10 / 0.25)[\text { POU 92]; } \mathbf{0 . 1 9} \pm 0.05(0.12 / 0.25)[\text { ORT 95]; } \mathbf{0 . 1 5} \pm 0.03(0.10 / 0.24) \\
{[\text { PEZ 95A]; 0.17 } \pm 0.05(0.09 / 0.25)[\text { MAT 98]; }(0.10 / 0.35)[\text { BOG } 99] ; \mathbf{0 . 1 6} \pm 0.04[\text { PER 00] }}\end{array}$ \\
\hline
\end{tabular}




\begin{tabular}{|c|c|}
\hline Specific rotation $[\alpha]_{\mathrm{D}}^{20}$ & $\mathbf{- 2 . 2 5}$ [JUA 92]; -2.78 $\pm 2.99(-8.45 / 4.67)[\mathrm{PEZ} \mathrm{95A];} \mathrm{-6.9 \pm 2.5} \mathrm{[PER} \mathrm{00]}$ \\
\hline $\mathrm{pH}$ & $\begin{array}{l}\text { 3.8 (3.5/4.1) [ITA 75, SAU 82A, GON 83]; 3.7 } \pm 0.03 \text { [PEZ 90]; 3.9 } \pm 0.2(3.2 / 4.1)[P O U \\
92] ; \text { 3.7 (3.4/3.9) [PEZ 95A]; (3.7/4.5) [RUS 97]; 3.9 } \pm 0.18 \text { (3.68/4.20) [MAT 98]; } \\
\text { 3.8 } \pm 0.2 \text { [PER 00] }\end{array}$ \\
\hline Free acidity $(\mathrm{meq} / \mathrm{kg})$ & $\begin{array}{l}\text { 7.4*(4.0/11.0) [ITA 75, SAU 82A, GON 83]; 16.7 } \pm 0.43 \text { [PEZ 90]; 16.2 } \pm 2.2(10.5 / 20.0) \\
\text { [PEZ 95A]; 20.0 } \pm 0.7 \text { [MEN 98]; (11/22) [RUS 97]; 15.7 } \pm 4.1 \text { [PER 00] }\end{array}$ \\
\hline Lactones (meq/kg) & $\begin{array}{l}\text { 6.2*(1.0/10.4) [ITA 75, SAU 82A, GON 83]; 0.93 } \pm 0.20 \text { [PEZ 90]; 1.1 } \pm 1.3(0 / 6.1)[\text { PEZ } \\
95 \mathrm{~A}] ; \mathbf{2 . 1} \pm 1.7 \text { [PER 00] }\end{array}$ \\
\hline Total acidity $(\mathrm{meq} / \mathrm{kg})$ & $\begin{array}{l}\text { 13.6*(8.7/19.1) [ITA 75, SAU 82A, GON 83]; 17.6 } \pm 0.53 \text { [PEZ 90]; 13.6 } \pm 2.8(9.0 / 19.0) \\
{[\text { POU 92]; 17.2 } \pm 2.45(10.5 / 21.5)[\text { PEZ 95A]; 17.7 } \pm 4.6[\text { PER 00] }}\end{array}$ \\
\hline Water $\mathrm{g} / 100 \mathrm{~g}$ & $\begin{array}{l}<\mathbf{1 7 . 5} \text { [ITA 75, GON 83]; 19.2 } \pm 1.8(16.6 / 21.4) \text { [SER 87]; 16.6 } \pm 0.18 \text { [PEZ 90]; 17.6 } \pm 1.2 \\
(16.0 / 20.0) \text { [POU 92]; 16.8 } \pm 0.73(15.4 / 18.3) \text { [PEZ 95A]; 15.2 } \pm 0.02 \text { [MEN 98]; }(14.5 / \\
\text { 16.5) [RUS 97]; 19.1 } \pm 1.29(17.3 / 20.8) \text { [MAT 98]; 16.5 } \pm 1.1 \text { [PER 00] }\end{array}$ \\
\hline Diastase (DN) & $\begin{array}{l}<\mathbf{1 0}(10 / 20)[\text { ITA } 75, \text { GON } 83] ; \mathbf{3 0 . 9} \pm 11.4(16.7 / 42.9) \text { [SER 87]; 19.0 } \pm 6.8(10.0 / 36.0) \\
\text { [POU 92]; 18.0 } \pm 4.88(10 / 29) \text { [PEZ 95A]; 8.2 } \pm 0.6 \text { [MEN 98]; (3.3/10.6) [RUS 97]; } \\
\text { 9.1 } \pm 2.2 \text { [PER 00] }\end{array}$ \\
\hline Invertase (U/kg) & 61.0 $\pm 15.4(28.6 / 86.7)$ [PER 99]; 60.2 \pm 16.9 [PER 00]; 105.8 \pm 42.1 (44.1/176.3) [SER 00] \\
\hline Proline (mg/kg) & 320.0 \pm 70.0 [PER 00] \\
\hline Fructose $(g / 100 \mathrm{~g})$ & $\begin{array}{l}\text { 42.8**(38.8/45.0) [ITA 75, SAU 82A, GON 83]; (39.0/41.3) [GON 79]; 35.3 } \pm 1.2(33.4 / \\
\text { 37.5) [SER 87]; 36.1 [JUA 92]; 36.4 } \pm 0.9(32.8 / 39.0) \text { [POU 92]; 36.7 } \pm 2.3(33.4 / 40.5) \\
\text { [ORT 95]; 36.6 } \pm 2.43(31.9 / 42.5) \text { [PEZ 95A]; 36.2 } \pm 1.6 \text { (33.7/40.1) [MAT 97]; 37.9 } \pm 1.1 \\
\text { [MEN 98, MAT 98]; 38.5 } \pm 1.1 \text { [PER 00] }\end{array}$ \\
\hline Glucose $(\mathrm{g} / 100 \mathrm{~g})$ & $\begin{array}{l}\text { 39.3**(35.5/41.8) [ITA 75, SAU 82A, GON 83]; (36.9/38.5) [GON 79]; 29.5 } \pm 1.3(27.8 / \\
32.6) \text { [SER 87]; 30.8 [JUA 92]; 32.4 } \pm 0.9(29.0 / 35.0) \text { [POU 92]; 28.5 } \pm 1.4(26.3 / 30.1) \\
\text { [ORT 95]; 31.2 } 2.3(26.3 / 36.0) \text { [PEZ 95A]; 31.2 } \pm 2.3 \text { (28.6/37.0) [MAT 97]; 31.6 } \pm 3.2 \\
\text { [MEN 98, MAT 98]; 33.7 } \pm 1.3 \text { [PER 00] }\end{array}$ \\
\hline Sucrose $(g / 100 \mathrm{~g})$ & $\begin{array}{l}\text { 3.86**(0.90/12.5) [ITA 75, SAU 82A, GON 83]; 1.1 } \pm 1.11(0.05 / 3.7) \text { [SER 87]; 2.2 [JUA } \\
\text { 92]; 2.7 } \pm 1.9(0.6 / 6.8) \text { [POU 92]; 4.2 } \pm 3.2(1.0 / 8.7) \text { [ORT 95]; 1.97 } \pm 1.39(0.07 / 5.85) \\
\text { [PEZ 95A]; 1.6 } \pm 2.2(0.045 / 5.7) \text { [MAT 97, MAT 98]; (2.9***/11.9) [RUS 97]; }(0.0 / 4.6) \\
\text { [BOG 99]; 0.3 } \pm 0.4 \text { [PER 00] }\end{array}$ \\
\hline $\mathrm{F}+\mathrm{G}(\mathrm{g} / 100 \mathrm{~g})$ & $(64.3 * * * * / 71.6)$ [RUS 97]; (64.8/84.1) [BOG 99]; 72.2 \pm 1.9 [PER 00] \\
\hline $\mathrm{F} / \mathrm{G}$ ratio & $\begin{array}{l}\text { 1.08 (1.02/1.13) [ITA 75, SAU 82A, GON 83]; 1.12 } \pm 0.03(1.08 / 1.21) \text { [POU 92]; } \\
\mathbf{1 . 1 7} \pm 0.1(0.99 / 1.40) \text { [MAT 97]; 1.20 [MEN 98, MAT 98]; 1.14 } \pm 0.05 \text { [PER 00] }\end{array}$ \\
\hline $\mathrm{G} / \mathrm{W}$ ratio & $\mathbf{1 . 6 4} \pm 0.14(1.43 / 1.89)[$ MAT 97, MAT 98]; 2.03 \pm 0.11 [PER 00] \\
\hline $\begin{array}{l}* \text { pH at equivalent poin } \\
* * \% \text { of dry matter } \\
* * * \text { apparent sucrose } \\
* * * * \text { reducing sugars }\end{array}$ & \\
\hline
\end{tabular}




\section{TARAXACUM}

\begin{tabular}{|c|c|}
\hline $\mathrm{PG} / 10 \mathrm{~g}$ honey $\cdot 10^{3}$ & $<60$ [PER 00] \\
\hline$\%$ Specific pollen & (15/60) [BOG 90]; low, very low [BRU 92]; 29 \pm 15 [BOG 97]; (5/30) [PER 00] \\
\hline Colour (mm Pfund) & $\mathbf{5 1} \pm 8(41 / 62)[$ BRU 92]; 54 \pm 11 (41/71) [PER 95A]; 56.8 \pm 9.8 [PER 00] \\
\hline $\begin{array}{l}\text { Electrical conductivity } \\
(\mathrm{mS} / \mathrm{cm})\end{array}$ & $\begin{array}{l}\text { 0.56 [VOR 64]; (0.50/0.65) [BOG 90]; 0.42 } \pm 0.09 \text { (0.29/0.52) [BRU 92]; } \mathbf{0 . 5 2} \pm 0.04 \\
(0.46 / 0.59) \text { [PER 95A, PER 00]; 0.59 } \pm 0.05[\text { BOG 97]; (0.29/0.65) [BOG 99] }\end{array}$ \\
\hline Specific rotation $[\alpha]_{\mathrm{D}}^{20}$ & 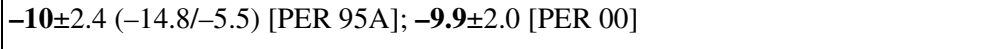 \\
\hline $\mathrm{pH}$ & 4.4 $\pm 0.3(4.1 / 5.0)[B R U ~ 92] ; \mathbf{4 . 5} \pm 0.2(4.1 / 5.0)$ [PER 95A, PER 00]; 4.3 \pm 0.17 [BOG 97] \\
\hline Free acidity (meq/kg) & $\mathbf{9 . 9} \pm 1.8(8.0 / 13.0)[\mathrm{BRU} 92] ; \mathbf{1 1 . 7} \pm 2.8[$ PER 00] \\
\hline Lactones (meq/kg) & 1.9 \pm 1.8 [PER 00] \\
\hline Total acidity (meq/kg) & 13.2 $\pm 3.9(7.4 / 24.7)[$ PER 95A]; 12.3 \pm 2.4 [PER 00] \\
\hline Water $\mathrm{g} / 100 \mathrm{~g}$ & $\begin{array}{l}(14.4 / 17.2)[\text { BOG 90]; 16.3 } \pm 0.7(15.0 / 17.5)[B R U ~ 92] ; 16.9 \pm 0.9(<18.5)[\text { PER 95A]; } \\
\text { 17.1 } \pm 0.7 \text { [PER 00] }\end{array}$ \\
\hline Diastase (DN) & 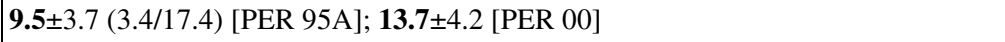 \\
\hline Invertase (U/kg) & 75.7 $\pm 21.3(39.7 / 106.5)[$ PER 99]; 102.8 \pm 26.4 [PER 00] \\
\hline Proline $(\mathrm{mg} / \mathrm{kg})$ & 340 \pm 70 [PER 00] \\
\hline Fructose $(\mathrm{g} / 100 \mathrm{~g})$ & 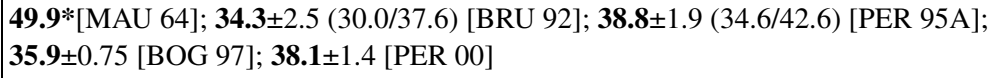 \\
\hline Glucose $(\mathrm{g} / 100 \mathrm{~g})$ & $\begin{array}{l}\text { 40.1*[MAU 64]; (32.2/37.1) [BOG 90]; 36.2 } \pm 2.4(33.6 / 41.0) \text { [BRU 92]; 39.1 } \pm 2.5 \\
\text { (34.5/43.5) [PER 95A]; 34.5 } \pm 1.37 \text { [BOG 97]; 38.4 } \pm 2.0 \text { [PER 00] }\end{array}$ \\
\hline Sucrose $(\mathrm{g} / 100 \mathrm{~g})$ & $\begin{array}{l}\text { 6.3*[MAU 64]; (0.1/0.6) [BOG 90]; 1.2 } \pm 1.5 \text { (tr./0.5) [PER 95A]; 0.37 } \pm 0.37 \text { [BOG 97]; } \\
(0.0 / 5.0) \text { [BOG 99]; 0.2 } \pm 0.6 \text { [PER 00] }\end{array}$ \\
\hline $\mathrm{F}+\mathrm{G}(\mathrm{g} / 100 \mathrm{~g})$ & 77.9 \pm 3.7 (70.3/84.2) [PER 95A]; (67.4/84.2) [BOG 99]; 76.5 \pm 3.0 [PER 00] \\
\hline F/G ratio & $\begin{array}{l}\mathbf{0 . 9 5} \pm 0.07(0.84 / 1.06)[\mathrm{BRU} 92] ; \mathbf{0 . 9 9} \pm 0.04(0.90 / 1.10)[\mathrm{PER} 95 \mathrm{~A}] ; \mathbf{1 . 1 1} \pm 0.08[\mathrm{BOG} \\
97] ; \mathbf{0 . 9 9} \pm 0.04[\text { PER 00] }\end{array}$ \\
\hline $\mathrm{G} / \mathrm{W}$ ratio & $\mathbf{2 . 2 7} \pm 0.12(2.03 / 2.49)[$ PER 95A]; 2.24 \pm 0.13 [PER 00] \\
\hline
\end{tabular}

\section{THYMUS}

\begin{tabular}{|c|c|}
\hline PG/10 g honey $\cdot 10^{3}$ & $<20$ [PER 00] \\
\hline$\%$ Specific pollen & $>26$ [DRI 95]; >15 [PER 00]; >18 [TSI 00] \\
\hline Colour (mm Pfund) & $52 \pm 16(27 / 83)[\mathrm{PER} 95 \mathrm{~A}] ; 49.7 \pm 11.6$ [PER 00] \\
\hline $\begin{array}{l}\text { Electrical } \\
\text { conductivity }(\mathrm{mS} / \mathrm{cm})\end{array}$ & $\begin{array}{l}(0.28 * / 0.74)(0.24 * * / 0.77)[\text { DRI 95]; } 0.38 \pm 0.04(0.32 / 0.49) \text { [PER 95A]; } 0.42 \pm 0.94(0.25 / \\
0.52) \text { [THR 95]; 0.42 } \pm 1.06(0.22 / 0.64) \text { [KAR 97]; }(0.24 / 0.72) \text { [BOG 99]; } 0.39 \pm 0.05 \\
\text { [PER 00]; } 0.39(0.25 / 0.50) \text { [TSI 00] }\end{array}$ \\
\hline
\end{tabular}




\begin{tabular}{|c|c|}
\hline Specific rotation $[\alpha]_{\mathrm{D}}^{20}$ & $-20.0 \pm 2.4(-24.5 /-17.0)[$ PER $95 \mathrm{~A}] ;-20.3 \pm 2.2$ [PER 00] \\
\hline $\mathrm{pH}$ & $\begin{array}{l}(2.2 * / 4.2)(2.7 * * / 4.2) \text { [DRI 95]; } 3.8 \pm 0.1(3.6 / 4.0) \text { [PER 95A, PER 00]; } 3.7 \pm 0.1(3.5 / 4.1) \\
\text { [THR 95]; } 3.4 \pm 0.4(2.2 / 4.1) \text { [KAR 97] }\end{array}$ \\
\hline Free acidity $(\mathrm{meq} / \mathrm{kg})$ & $37.1 \pm 6.1[$ PER 00] \\
\hline Lactones (meq/kg) & $2.7 \pm 2.5$ [PER 00] \\
\hline Total acidity (meq/kg) & $41.1 \pm 4.9(32.5 / 57.2)$ [PER 95A]; 39.8 \pm 5.2 [PER 00] \\
\hline Water $(\mathrm{g} / 100 \mathrm{~g})$ & $\begin{array}{l}(15.0 * / 20.3)(14.9 * * / 19.7)[\text { DRI 95]; } 16.5 \pm 1.3(<18.5) \text { [PER 95A]; } 16.1 \pm 0.51(15.4 / \\
\text { 17.0) [THR 95]; 17.1 } \pm 0.92(14.9 / 20.3) \text { [KAR 97]; 16.6 } \pm 1.1 \text { [PER 00]; } 15.7(14.7 / 17.9) \\
\text { [TSI 00] }\end{array}$ \\
\hline Diastase (DN) & 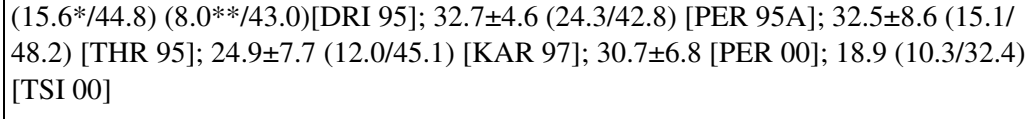 \\
\hline Invertase $(\mathrm{U} / \mathrm{kg})$ & $\begin{array}{l}35.3 \pm 9.0(23.5 / 54.4) \text { [KAR 97]; 135.1 } \pm 25.7(101.4 / 196.8) \text { [PER 99]; 136.6 } \pm 25.7 \text { [PER } \\
00] ; 112.4(30.8 / 213.0) \text { [TSI 00] }\end{array}$ \\
\hline Proline $(\mathrm{mg} / \mathrm{kg})$ & $\begin{array}{l}\text { 790 } \pm 232(596 / 1205) \text { [THR 95]; 893 } \pm 255 \text { (525/1311) [KAR 97]; 930 } \pm 110 \text { [PER 00]; } \\
1105 \text { (306/1873) [TSI 00] }\end{array}$ \\
\hline Fructose $(g / 100 g)$ & $42.6 \pm 2.5(38.0 / 49.2)$ [PER 95A, PER 00] \\
\hline Glucose $(g / 100 \mathrm{~g})$ & 30.2 $\pm 1.4(26.8 / 33.8)$ [PER 95A, PER 00]; (26.3/32.6) [MAN 01] \\
\hline Sucrose $(g / 100 \mathrm{~g})$ & $0.1 \pm 0.1(0 / 0.6)$ [PER 95A, PER 00]; (0.0/0.6) [BOG 99] \\
\hline $\mathrm{F}+\mathrm{G}(\mathrm{g} / 100 \mathrm{~g})$ & $72.8 \pm 3.1(68.0 / 80.3)$ [PER 95A, PER 00]; (64.0/80.3) [BOG 99] \\
\hline $\mathrm{F} / \mathrm{G}$ ratio & $1.41 \pm 0.10(1.23 / 1.58)$ [PER 95A, PER 00]; (1.27/1.49) [MAN 01] \\
\hline $\mathrm{G} / \mathrm{W}$ ratio & $1.86 \pm 0.18(1.52 / 2.25)$ [PER 95A, PER 00] \\
\hline
\end{tabular}

\section{TILIA}

\begin{tabular}{|c|c|}
\hline $\mathrm{PG} / 10 \mathrm{~g}$ honey $\cdot 10^{3}$ & $<20$ [PER 95A]; 11 $\pm 5.6(2 / 24)$ [PER 00] \\
\hline$\%$ Specific pollen & variable, very low [PER 95A, PER 00]; (21/38) [IVA 02] \\
\hline Colour (mm Pfund) & 52 $\pm 25(11 / 71)$ [BRU 92]; 43 \pm 17 (11/71) [PER 95A]; 35.4 \pm 12.6 [PER 00] \\
\hline $\begin{array}{l}\text { Electrical conductivity } \\
(\mathrm{mS} / \mathrm{cm})\end{array}$ & $\begin{array}{l}\mathbf{0 . 2 9} \text { [VOR 64]; (0.64/0.87) [RAV 75]; } \mathbf{0 . 6 4}(0.49 / 0.76)[\text { KRA 91]; } \mathbf{0 . 7 8} \pm 0.16(0.54 / \\
0.96)[\text { BRU 92]; } \mathbf{0 . 6 7} \pm 0.12(0.42 / 0.99)[\mathrm{PER} 95 \mathrm{~A}] ; \mathbf{0 . 8 1} \pm 0.08(0.70 / 0.91) \text { [GOL 99]; } \\
\mathbf{0 . 6 4} \pm 0.10[\mathrm{PER} 00]\end{array}$ \\
\hline Specific rotation $[\alpha]_{D}^{20}$ & $\mathbf{- 1 2 . 5} \pm 2.1(-18.0 /-8.0)[$ PER 95A]; - $\mathbf{1 1 . 8} \pm 2.4[$ PER 00] \\
\hline $\mathrm{pH}$ & $\begin{array}{l}\text { (4.05/4.91) [RAV 75]; } \mathbf{4 . 4}(4.2 / 4.7) \text { [KRA 91]; } \mathbf{4 . 5} \pm 0.3(4.2 / 5.1) \text { [BRU 92]; } 4.4 \pm 0.3(3.9 / \\
5.2)[\text { PER 95A]; 4.1 } \pm 0.35 \text { (3.9/4.8) [GOL 99]; } \mathbf{4 . 3} \pm 0.2 \text { [PER 00] }\end{array}$ \\
\hline
\end{tabular}




\begin{tabular}{|c|c|}
\hline Free acidity $(\mathrm{meq} / \mathrm{kg})$ & $\begin{array}{l}\text { 23.2 (14.5/35.1) [KRA 91]; 24.4 } \pm 10.1 \text { (13.0/35.0) [BRU 92]; 25.0 } \pm 5.1(16.0 / 30.0) \\
\text { [GOL 99]; 22.1 } \pm 8.6 \text { [PER 00]; (16.5/31.0) [IVA 02] }\end{array}$ \\
\hline Lactones (meq/kg) & 7.7 (5.2/9.2) [KRA 91]; 2.1 \pm 1.1 [PER 00] \\
\hline Total acidity (meq $/ \mathrm{kg})$ & 30.9 (20.1/44.2) [KRA 91]; 24.6 \pm 8.7 (8.5/38.1) [PER 95A]; 24.2 \pm 8.8 [PER 00] \\
\hline Water $(g / 100 \mathrm{~g})$ & $\begin{array}{l}(17.0 / 20.4) \text { [RAV 75]; 20.1 (17.4/21.3) [KRA 91]; 17.6 } \pm 1.2 \text { (16.3/18.9) [BRU 92]; } \\
\text { 16.9 } \pm 0.8(<18.5) \text { [PER 95A]; 16.6 } \pm 1.34 \text { (14.9/18.1) [GOL 99]; 16.8 } \pm 0.8 \text { [PER 00]; } \\
(16.7 / 18.8) \text { [IVA 02] }\end{array}$ \\
\hline Diastase (DN) & $\begin{array}{l}\text { 20.5 (13.9/29.4) [KRA 91]; 17.9 } \pm 5.3 \text { (9.6/34.3) [PER 95A]; 13.2 } \pm 1.94(10.6 / 15.4) \\
\text { [GOL 99]; 17.7 } \pm 3.7 \text { [PER 00]; (10.4/17.7) [IVA 02] }\end{array}$ \\
\hline Invertase (U/kg) & 157.2 [KRA 91]; 94.0 \pm 27.9 (49.2/145.4) [PER 99]; 94.0 \pm 30.1 [PER 00] \\
\hline Proline $(\mathrm{mg} / \mathrm{kg})$ & $\mathbf{4 2 4 . 3}$ (243.8/568.3) [KRA 91]; $\mathbf{4 2 0 . 0 \pm 9 0 . 0 ~ [ P E R ~ 0 0 ] ; ~} \mathbf{4 6 9}$ (373/640) [IVA 02] \\
\hline Fructose $(g / 100 \mathrm{~g})$ & $\begin{array}{l}\text { 38.9 (35.9/41.0) [KRA 91]; 38.6 } \pm 1.7(37.5 / 40.6) \text { [BRU 92]; 38.3 } \pm 4.0(30.2 / 47.2)[\mathrm{PER} \\
95 \mathrm{~A}] ; \mathbf{3 9 . 1} \text { [IVA 97]; 39.9 } \pm 2.89(35.7 / 43.0) \text { [GOL 99]; 39.5 } \pm 2.8[\mathrm{PER} 00]\end{array}$ \\
\hline Glucose $(\mathrm{g} / 100 \mathrm{~g})$ & $\begin{array}{l}\text { 34.6 (31.7/35.9) [KRA 91]; 32.3 } \pm 1.3(31.1 / 34.2) \text { [BRU 92]; 30.3 } \pm 2.2(25.7 / 34.8)[\mathrm{PER} \\
\text { 95A]; 27.3 [IVA 97]; 31.6 } \pm 1.4(29.0 / 33.1) \text { [GOL 99]; 30.7 } \pm 2.1 \text { [PER 00] }\end{array}$ \\
\hline Sucrose $(g / 100 \mathrm{~g})$ & $\begin{array}{l}\mathbf{0 . 3 8}(0.22 / 0.54) \text { [KRA 91]; } \mathbf{0 . 1 0} \pm 0.2(0.0 / 0.4) \text { [BRU 92]; } \mathbf{0 . 1 0} \pm 0.1(0 / 0.5) \text { [PER 95A, } \\
\text { PER 00]; 1.23 [IVA 97]; } \mathbf{0 . 5 7} \pm 0.21(0.42 / 0.72) \text { [GOL 99] }\end{array}$ \\
\hline $\mathrm{F}+\mathrm{G}(\mathrm{g} / 100 \mathrm{~g})$ & $\begin{array}{l}\text { 73.5 (71.4/76.5) [KRA 91]; 68.7 } \pm 5.2(58.9 / 77.7)[\text { PER 95A]; 70.6 } \pm 2.14(66.1 / 73.03) \\
\text { [GOL 99]; 70.2 } \pm 3.9 \text { [PER 00] }\end{array}$ \\
\hline $\mathrm{F} / \mathrm{G}$ ratio & $\begin{array}{l}\text { 1.20 } \pm 0.04(1.16 / 1.25)[\text { BRU 92]; 1.27 } \pm 0.13(1.00 / 1.60)[\text { PER 95A]; 1.24 } \pm 0.14(1.10 / \\
1.48)[\text { GOL 99]; 1.29 } \pm 0.11 \text { [PER 00] }\end{array}$ \\
\hline $\mathrm{G} / \mathrm{W}$ ratio & 1.79 $\pm 0.16(1.50 / 2.00)[$ PER $95 \mathrm{~A}] ; \mathbf{1 . 8 3} \pm 0.14$ [PER 00] \\
\hline
\end{tabular}

\section{HONEYDEW HONEY}

\begin{tabular}{|c|c|}
\hline PE/10 g honey & variable $(<300,000)[$ PER 00] \\
\hline HDE/PG ratio & variable [PER 95A] \\
\hline Colour (mm Pfund) & $\begin{array}{l}\text { 86 } \pm 14(51 / 110)[\text { BRU 92]; } 119(83 / 140) \text { [MAT 92]; 80 } \pm 7 \text { (62/106) [POU 92]; 99 } \pm 16(83 / \\
\text { 130) [PER 95A]; 98.4 } \pm 15.2 \text { [PER 00] }\end{array}$ \\
\hline $\begin{array}{l}\text { Electrical conductivity } \\
(\mathrm{mS} / \mathrm{cm})\end{array}$ & 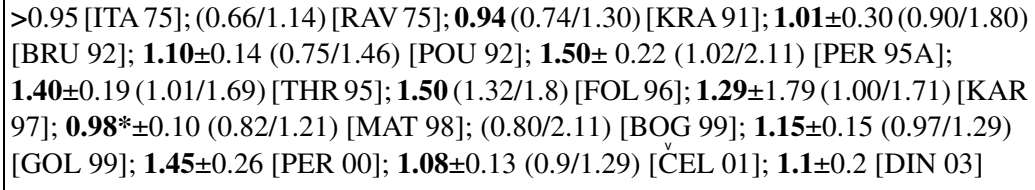 \\
\hline Specific rotation $[\alpha]_{D}^{20}$ & 14.0 $\pm 5.0(6.0 / 29.7)[$ PER 95A]; 14.3 \pm 5.7 [PER 00]; $\mathbf{4 . 2} \pm 1.3$ [DIN 03] \\
\hline $\mathrm{pH}$ & 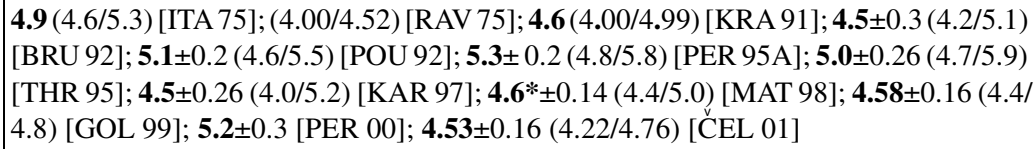 \\
\hline
\end{tabular}




\begin{tabular}{|c|c|}
\hline Free acidity (meq/kg) & $\begin{array}{l}\text { 19.8**(14.4/26.2) [ITA 75]; 33.1 (23.2/47.5) [KRA 91]; 31.3 } \pm 6.4 \text { (22.0/39.0) [BRU 92]; } \\
\text { 25.7 (22.5/28.9) [KAR 97]; 26.5 } \pm 6.0 \text { [PER 00] }\end{array}$ \\
\hline Lactones (meq/kg) & $\begin{array}{l}\text { 3.0**(1.0/8.5) [ITA 75]; 6.5 (4.5/9.8) [KRA 91]; 5.6 (5.11/6.1) [KAR 97]; 1.9 } \pm 1.4[\text { PER } \\
00]\end{array}$ \\
\hline Total acidity (meq/kg) & $\begin{array}{l}\mathbf{2 2 . 9} * *(15.8 / 30.6) \text { [ITA } 75] ; 39.7(29.7 / 57.2) \text { [KRA 91]; 23.0 } \pm 4.3(14.3 / 36.5) \text { [POU 92]; } \\
\mathbf{2 5 . 4} \pm 5.8(17.3 / 39.2) \text { [PER 95A]; 31.3(28.6/34.1) [KAR 97]; 17.4 } \pm 4.9(10.3 / 24.8) \text { [GOL } \\
\text { 99]; 28.4 } \pm 6.7 \text { [PER 00]; 32.7 } \pm 10.6(14.3 / 53.5) \text { [CEL 01] }\end{array}$ \\
\hline Water $(g / 100 \mathrm{~g})$ & $\begin{array}{l}<\mathbf{1 8} \text { [ITA 75]; }(17.8 / 20.5) \text { [RAV 75]; 19.7 (18.5/21.9) [KRA 91]; 17.6 } \pm 1.2(15.3 / 19.2) \\
\text { [BRU 92]; 16.3 } \pm 1.1(14.0 / 18.8) \text { [POU 92]; 16.1 } \pm 1.0(<18) \text { [PER 95A]; 15.2 } \pm 1.5(13.0 / \\
\text { 18.5) [THR 95]; 16.3 } \pm 0.82(14.3 / 18.2) \text { [KAR 97]; 15.8* } \pm 0.43(14.6 / 16.4) \text { [MAT 98]; } \\
\mathbf{1 6 . 6} \pm 2.06(14.5 / 19.8) \text { [GOL 99]; 15.5 } \pm 0.9 \text { [PER 00]; 15.6 } \pm 1.7(13.0 / 19.8) \text { [CEL 01]; } \\
\mathbf{1 7 . 2} \pm 1.2 \text { [DIN 03] }\end{array}$ \\
\hline Diastase (DN) & $\begin{array}{l}>20 \text { [ITA 75]; } 23.2(8.3 / 38.5) \text { [KRA 91]; 22.6 } \pm 6.7(10.9 / 34.1) \text { [PER 95A]; 18.5 } \pm 5.46 \\
(10.4 / 29.6)[\text { THR 95]; 18.8 } \pm 4.62(11.3 / 35.6) \text { [KAR 97]; 16.5 } \pm 3.2(12.3 / 21.1) \text { [GOL } 99] ; \\
\text { 23.4 } \pm 5.8 \text { [PER 00] }\end{array}$ \\
\hline Invertase (U/kg) & $\begin{array}{l}\text { 39.7 } \pm 8.8(27.2 / 58.8) \text { [KAR 97]; 175.5 } \pm 22.0(131.5 / 224.8) \text { [PER 99]; 172.6 } \pm 28.6 \text { [PER } \\
00] ; \mathbf{2 2 3 . 9} \pm 43.5 \text { [DIN 03] }\end{array}$ \\
\hline Proline (mg/kg) & $\begin{array}{l}\mathbf{6 0 6}(387 / 1113) \text { [KRA 91]; 390 } \pm 194 \text { (290/580) [THR 95]; } 563 \text { (487/608) [FOL 96]; } \\
\mathbf{5 9 2} \pm 140 \text { (368/840) [KAR 97]; } \mathbf{5 6 4 . 5} \pm 23.3 \text { [DIN 03] }\end{array}$ \\
\hline Fructose $(\mathrm{g} / 100 \mathrm{~g})$ & $\begin{array}{l}\text { 37.1*** (33.3/41.5) [ITA 75]; 32.3 (28.3/37.2) [BOG 88]; 34.0 (29.9/35.9) [KRA 91]; } \\
\mathbf{3 6 . 8} \pm 2.9(33.2 / 41.4) \text { [BRU 92]; 31.3 } \pm 1.8(28.0 / 40.2) \text { [POU 92]; 31.8 } \pm 2.8(24.5 / 35.8) \\
\text { [PER 95A]; (32.0/34.8) [IVA 97]; 34.3* } \pm 1.1(32.6 / 35.9) \text { [MAT 97, MAT 98]; 34.2 } \pm 2.9 \\
\text { (30.8/37.6) [GOL 99]; 31.5 } \pm 2.6 \text { [PER 00] }\end{array}$ \\
\hline Glucose $(\mathrm{g} / 100 \mathrm{~g})$ & 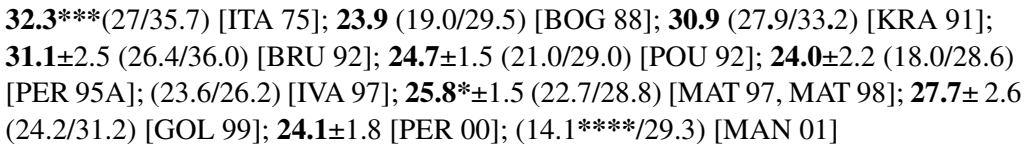 \\
\hline Sucrose (g/100 g) & $\begin{array}{l}\mathbf{0 . 6 4} * * *(0.10 / 1.20)[\text { ITA } 75] ; \mathbf{0 . 5}(0.1 / 1.0) \text { [BOG } 88] ; \mathbf{0 . 9 8}(0.35 / 4.41) \text { [KRA 91]; } \\
\mathbf{0 . 2} \pm 0.4(0.0 / 1.8) \text { [BRU 92]; } \mathbf{1 . 0} \pm 1.0(0.1 / 3.6) \text { [POU 92]; 0.4 } \pm 0.4 \text { (tr./1.8) [PER 95A]; } \\
\mathbf{0 . 2 1} * \pm 0.17(0.02 / 0.75) \text { [MAT 97, MAT 98]; (0.0/4.8) [BOG 99]; } \mathbf{0 . 4} \pm 0.4 \text { [PER 00]; } \\
\mathbf{2 . 5 4} \pm 1.9(0.10 / 5.69) \text { [CEL 01] }\end{array}$ \\
\hline $\mathrm{F}+\mathrm{G}(\mathrm{g} / 100 \mathrm{~g})$ & $\begin{array}{l}\text { 67.9 [BRU 92]; 56.0 [POU 92]; 55.8 } \pm 4.6 \text { (42.5/63.0) [PER 95A]; (57/58.2) [IVA 97]; } \\
(45.1 / 71.8) \text { [BOG 99]; 61.9 } \pm 4.8(56.7 / 67.3) \text { [GOL 99]; 55.7 } \pm 3.8 \text { [PER 00] }\end{array}$ \\
\hline $\mathrm{F} / \mathrm{G}$ ratio & 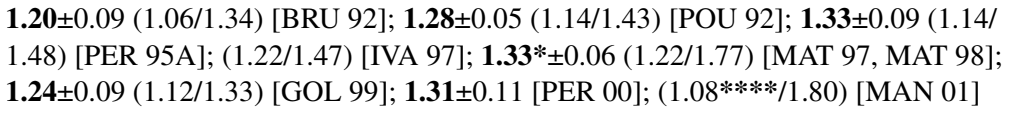 \\
\hline $\mathrm{G} / \mathrm{W}$ ratio & $\begin{array}{l}\mathbf{1 . 5 0} \pm 0.12(1.20 / 1.72)[\text { PER } 95 \mathrm{~A}] ; \mathbf{1 . 6 3}^{*} \pm 0.10(1.45 / 1.77)[\text { MAT 97, MAT 98]; } \\
\mathbf{1 . 5 5} \pm 0.13[\text { PER 00] }\end{array}$ \\
\hline \multicolumn{2}{|c|}{$\begin{array}{l}\text { * honeydew honey of Quercus spp } \\
\text { ** pH at equivalent point } \\
\text { ***\% of dry matter } \\
\text { **** honeydew honey of pine and of fir trees }\end{array}$} \\
\hline
\end{tabular}


METCALFA HONEYDEW HONEY

\begin{tabular}{|c|c|}
\hline $\mathrm{PE} / 10 \mathrm{~g}$ honey & variable, $<300,000[$ PER 00] \\
\hline $\mathrm{HDE} / \mathrm{PG}$ ratio & $>3$ [PER 00] \\
\hline Colour (mm Pfund) & 98 $\pm 8(83 / 110)[\mathrm{PER} 95 \mathrm{~A}] ; \mathbf{1 0 2 . 4} \pm 6.8$ [PER 00] \\
\hline $\begin{array}{l}\text { Electrical } \\
\text { conductivity }(\mathrm{mS} / \mathrm{cm})\end{array}$ & $\mathbf{1 . 6 4} \pm 0.27(0.96 / 2.05)[\mathrm{PER} 95 \mathrm{~A}] ; \mathbf{1 . 7 4} \pm 0.19$ [PER 00]; 1.92 $\pm 0.32(1.26 / 2.38)$ [GOL 03] \\
\hline Specific rotation $[\alpha]_{\mathrm{D}}^{20}$ & 17 $\pm 7.4(2.5 / 30.0)[$ PER 95A]; 18.5 \pm 6.5 [PER 00] \\
\hline $\mathrm{pH}$ & 5.0 $\pm 0.4(4.3 / 5.9)[$ PER 95A]; 5.1 \pm 0.4 [PER 00]; 5.4 \pm 0.3 (4.9/6.3) [GOL 03] \\
\hline Free acidity $(\mathrm{meq} / \mathrm{kg})$ & 37.2 \pm 6.1 [PER 00]; 28.7 $\pm 4.6(18.5 / 38.0)$ [GOL 03] \\
\hline Lactones (meq/kg) & 4.1 \pm 1.3 [PER 00]; 1.6 $\pm 1.1(0.0 / 3.6)$ [GOL 03] \\
\hline Total acidity $(\mathrm{meq} / \mathrm{kg})$ & 40.6 $\pm 6.6(25.7 / 57.7)[\mathrm{PER} 95 \mathrm{~A}] ; \mathbf{4 1 . 3} \pm 6.5$ [PER 00]; 30.3 $\pm 5.1(18.5 / 40.8)$ [GOL 03] \\
\hline Water $(\mathrm{g} / 100 \mathrm{~g})$ & 16 $\pm 0.8(<18)[$ PER 95A]; 15.8 \pm 0.7 [PER 00]; 14.7 $\pm 0.76(13.5 / 16.4)$ [GOL 03] \\
\hline Diastase (DN) & 31.9 $\pm 9.3(14.6 / 62.1)$ [PER 95A]; 34.2 \pm 7.5 [PER 00]; 22.9 \pm 7.3 (8.9/42.9) [GOL 03] \\
\hline Invertase $(\mathrm{U} / \mathrm{kg})$ & 171.9 $\pm 17.6(135.9 / 206.4)[$ PER 99]; 172.6 \pm 21.3 [PER 00] \\
\hline Proline $(\mathrm{mg} / \mathrm{g})$ & $\mathbf{5 2 0} \pm 170$ [PER 00]; 365 $\pm 100(200 / 636)$ [GOL 03] \\
\hline Fructose $(\mathrm{g} / 100 \mathrm{~g})$ & 31.9 $\pm 3.3(25.2 / 38.8)[$ PER 95A]; 31.7 \pm 3.3 [PER 00] \\
\hline Glucose $(g / 100 \mathrm{~g})$ & 23.7 $\pm 2.7(18.8 / 29.0)[$ PER 95A, PER 00] \\
\hline Sucrose $(g / 100 \mathrm{~g})$ & 0.1 \pm 0.1 (tr./0.4) [PER 95A, PER 00] \\
\hline $\mathrm{F}+\mathrm{G}(\mathrm{g} / 100 \mathrm{~g})$ & 55.6 $\pm 4.6(45.5 / 64.8)[$ PER 95A]; 55.3 \pm 4.7 [PER 00] \\
\hline F/G ratio & $\mathbf{1 . 3 6} \pm 0.19(0.87 / 1.76)[\mathrm{PER} 95 \mathrm{~A}] ; \mathbf{1 . 3 5} \pm 0.19$ [PER 00] \\
\hline G/W ratio & $\mathbf{1 . 4 8} \pm 0.15(1.22 / 1.88)[\mathrm{PER} 95 \mathrm{~A}] ; \mathbf{1 . 4 9} \pm 0.16$ [PER 00] \\
\hline
\end{tabular}

\section{REFERENCE LIST}

[BAC 65] Baculinschi H. (1965) Constatele fizicochimice si biologice la unele sorturi de miere, Lucr. Stiint. Stat. Cent. Seri. Apic. 5, 65-70 (quoted by Crane E., Walker P., Day R. (1984) Directory of important world honey sources, IBRA, London).

[BOG 88] Bogdanov S., Baumann E. (1988) Bestimmung von Honigzuckern mit HPLC, Mitt. Geb. Lebensm. Hyg. 79, 198-206.

[BOG 90] Bogdanov S. (1990) Les miels monofloraux en Suisse, J. Suisse Apic. 87, 15-21.

[BOG 97] Bogdanov S. (1997) Charakterisierung von Schweizer Sortenhonigen, Agrarforschung 4, 427-430.

[BOG 99] Bogdanov S., Lüllmann C., Martin P., von der Ohe W., Russmann H., Vorwohl G., Persano Oddo L., Sabatini A.G., Marcazzan G.L., Piro R., Flamini C., Morlot M., Lheritier J., Borneck R., Marioleas P., Tsigouri A., Kerkvliet J., Ortiz A., Ivanov T., D'Arcy B., Mossel B., Vit P. (1999)
Honey quality, methods of analysis and international regulatory standards, review of the work of the International Honey Commission, Mitt. Lebensm. Hyg. 90, 108-125.

[BRU 92] Bruneau E. (1992) personal communication.

[CAB 97] Cabrera Ruiz C., Montilla Gomez J., Hernandez Guerra E., Molins Marin J.L. (1997) Analyse physico-chimique des miels d'oranger commercialisés en Espagne, Bull. Tech. Apic. 24, 63-70.

[ČEL 01] Čelechovská O., Vorlová L. (2001) Groups of honey - Physicochemical properties and heavy metals, Acta Vet. Brno 70, 91-95.

[DIN 03] Dinkov D. (2003) A scientific note on the specific optical rotation of three honey types from Bulgaria, Apidologie 34, 319-320.

[DRI 95] Drimjias N., Karabournioti S. (1995) Les caractéristiques du miel de thym grec, Apiacta XXX, 33-39.

[DUS 67] Dustmann J.H. (1967) Messungen von Wasserstoffperoxid in Bienenhonig aus Edelkastanientracht, Z. Lebensm. Unters. Forsch. 134, 20. 
[ESP 81] Espada Herrero T. (1981) Composition chimique des miels monofloraux de Catalogne (Espagne), Proc. XXVIII Int. Beekeep. Congr. Acapulco, pp. 414-415.

[FAU 02] Faucon J.P., Martel A.C., Antinelli J.F., Clément M.C., Zeggane S., Cordella C., Davico R., Rognone C., Aurières C. (2002) Sondage sur la qualité des miels de lavande-lavandin, Bull. Tech. Apic. 29, 55-62.

[FOL 94] Foldházi G. (1994) Analysis and quantitation of sugars in honey of different botanical origin using HPLC, Acta Aliment. 23, 299-311.

[FOL 96] Foldházi G., Amtmann M., Fodor P., Ittzés A. (1996) The physico-chemical properties and composition of honeys of different botanical origin, Acta Aliment. 25, 237-256.

[GOL 99] Golob T., Plestenjak A. (1999) Quality of Slovene honey, Food Technol. Biotechnol. 37, 195-201.

[GOL 03] Golob T., Škrabanja V., Jamnik M., Bertoncelj J. (2003) Sensory and chemical characterisrtics of Metcalfa pruinosa honeydew honey, XXXVIII Int. Beekeep. Congr. Apimondia Ljubljana, CD rom, Abstract book, pp. 324-325.

[GON 79] Gonnet M. (1979) Application au miel d'une méthode de dosage par voie enzymatique des monosaccharides réducteurs, Apidologie 10,395401 .

[GON 83] Gonnet M. (1983) Le romarin, in: La fleur et l'abeille, Paris, Union Nationale de l'Apiculture Française, pp. 104-107.

[GON 87] Gonnet M. (1987) Caractéristiques, technologie et commercialisation des miels de colza et de tournesol, Abeilles et fleurs 367, 10-13.

[ITA 75] Institut technique de l'apiculture, Groupe de travail miel (1975) Caractéristiques de quelques miels monofloraux et miellats de la production française, XXV Congr. Int. Apiculture Apimondia Grenoble, pp. 541-548.

[IVA 78] Ivanov Ts. (1978) Composition and properties of Bulgarian honey, Beekeeping Experimental Station, Sofia, Bulgaria, 34 p. (quoted by Crane E., Walker P., Day R. (1984) Directory of important world honey sources, IBRA, London).

[IVA 97] Ivanov Ts. (1997) Determination of carbohydrates of honey by HPLC, J. Anim. Sci. 7-8, 108110.

[IVA 02] Ivanov Ts. (2002) personal communication.

[JAT 95] Jato Rodríguez V., Iglesias Fernández M.I., García Monjo J., Marsá Vilá M., Seijo Coello M.C. (1995) Caratteristiche melissopalinologiche e fisico-chimiche dei mieli di Rubus e di Castanea della provincia di Orense, Apicoltura 10, 11-22.

[JUA 92] Juan T., Conchello M.P., Tello M.L., Peréz Arquillé C., Herrera A. (1992) Rotación específica y espectro glucídico de mieles de Zaragoza, Alimentaria 232, 75-78.
[KAR 97] Karabournioti S., Drimjias N. (1997) Quelques caractéristiques physiques et chimiques des miels monofloraux de Grèce, Apiacta XXXII, 44-50.

[KRA 91] Krauze A., Zalewski R.I. (1991) Classification of honeys by principal component analysis on the basis of chemical and physical parameters, $\mathrm{Z}$. Lebensm. Unters. Forsch. 192, 19-23.

[MAN 01] Manikis I., Thrasyvoulou A. (2001) The relation of physicochemical characteristics of honey and the crystallization sensitive parameters, Apiacta $36,106-112$.

[MAT 92] Mateo Castro R., Jimenez Escamilla M., Bosch Reig F. (1992) Evaluation of the color of some Spanish unifloral honeys types as a characterization parameter, J. AOAC Int. 75, 537-542.

[MAT 97] Mateo Castro R., Bosch Reig F. (1997) Sugar profiles of Spanish unifloral honeys, Food Chem. $60,33-41$.

[MAT 98] Mateo R., Bosch-Reig F. (1998) Classification of Spanish unifloral honeys by discriminant analysis of electrical conductivity, color, water content, sugars and pH, J. Agric. Food Chem. 46 , $393-400$

[MAU 64] Maurizio A. (1964) Das Zuckerbild blutenreiner Sortenhonige, Ann. Abeille 7, 289-299 (quoted by Crane E., Walker P., Day R. (1984) Directory of important world honey sources, IBRA, London)

[MEN 98] Mendes E., Brojo Proença E., Ferreira I., Ferreira M.A. (1998) Quality evaluation of Portuguese honey, Carbohydr. Polym. 37, 219-223.

[MUR 76] Murko D., Pasic T., Ramic S. (1976) Istrazivanje sastava raznih vrsta pcelinjeg meda, Hemijska Ind. 30, 113-115 (quoted by Crane E. Walker P., Day R. (1984) Directory of important world honey sources, IBRA, London).

[ORT 95] Ortiz Valbuena A., Fernández Maeso M.S., Subrá Muñoz De La Torre E. (1995) Study of some physico-chemical parameters in honeys from La Alcarria (Spain), XXXIV Int. Beekeep. Congr. Apimondia Lausanne, pp. 321-333.

[PER 95A] Persano Oddo L., Piazza M.G., Sabatini A.G., Accorti M. (1995) Characterization of unifloral honeys, Apidologie 26, 453-465.

[PER 95B] Persano Oddo L., Piazza M.G., Zellini G. (1995) Caratteristiche cromatiche dei mieli uniflorali, Apicoltura 10, 109-120.

[PER 99] Persano Oddo L., Piazza M.G., Pulcini P. (1999) Invertase activity in honey, Apidologie 30, 57-65.

[PER 00] Persano Oddo L., Sabatini A.G., Accorti M., Colombo R., Marcazzan G.L., Piana M.L., Piazza M.G., Pulcini P. (2000) I mieli uniflorali italiani. Nuove schede di caratterizzazione, Ministero delle Politiche Agricole - Istituto Sperimentale Zoologia Agraria, Roma. 
[PEZ 90] Pérez C., Conchello P., Ariño A., Ucar A. Herrera A. (1990) Estudio de algunos parámetros físico-químicos en mieles monoflorales de Zaragoza, Alimentaria 213, 59-61.

[PEZ 95A] Pérez-Arquillué C., Conchello P., Ariño A., Juan T., Herrera A. (1995) Physicochemical attributes and pollen spectrum of some unifloral Spanish honeys, Food Chem. 54, 167-172.

[PEZ 95B] Pérez C., Conchello P., Ariño A., Juan T., Ucar A., Herrera A., Negueruela I. (1995) Una marca para la miel de romero, Vida Apic. 74, 55-57.

[POU 92] Pourtallier J. (1992) personal communication.

[PUJ 94] Pujolá M., Sanz A. (1994) Mieles de castaño y castaño y retama. Determinación de la acidez libre y otro parámetros físico-químicos, Vida Apic. 63, 56-60.

[RAV 75] Ravn V., Hammer B., Bartels H. (1975) An investigation of the sugar chemistry and an analysis of the pollen content in some types of Danish honeys, Tidssk. Planteavl 79, 13-36.

[RUS 97] Russo-Almeida P.A. (1997) Caracterização de alguns parâmetros químicos do mel da terra Quente Transmontana, O Apicoltor, 29-35.

[SAN 01] Sánchez M.D., Huidobro J.F., Mato I., Muniategui S., Sancho M.T. (2001) Correlation between proline content of honeys and botanical origin, Dtsch Lebensm. Rundsch. 97, 171-175.

[SAU 82A] Sauret J. (1982) El romero, Vida Apic. 1, 7-8.

[SAU 82B] Sauret J. (1982) Normas de calidad de la miel de espliego, Vida Apic. 2, 13-14.

[SER 87] Serra Bonvehí J., Pajuelo G., Gonell Galindo J. (1987) Composición, propriedades fisico-químicas y espectro polínico de algunas mieles monoflorales de España, Alimentaria 185, 61-84.

[SER 88A] Serra Bonvehí J. (1988) Propriétés physicochimiques, composition et spectre pollinique des miels de Lavandula latifolia Med. produits en Espagne, Sci. Aliment. 8, 295-307.

[SER 88B] Serra Bonvehí J. (1988) Determinación de antranilato de metilo en la miel de cítricos (Citrus sp.) del Levante Español, y su influencia en la actividad diastásica de la miel, Alimentaria 197, 3740.

[SER 88C] Serra Bonvehí J., Cañas Lloria S. (1988) Caratteristiche fisico chimiche, composizione e spettro pollinico del miele di Eucalipto (Eucalyptus spp.) prodotto in Spagna, Apicoltura 4, 59-81.
[SER 93] Serra Bonvehí J., Granados Tarrés E. (1993) Physicochemical properties, composition and pollen spectrum of ling heather (Calluna vulgaris (L.) Hull) honey produced in Spain, Apidologie 24, 586-596.

[SER 95] Serra Bonvehí J., Ventura Coll F. (1995) Characterization of Citrus honey (Citrus spp.) produced in Spain, XXXIV Int. Beekeep. Congr. Apimondia, Lausanne, pp. 358-364.

[SER 00] Serra Bonvehí J., Soliva Torrentó M., Muntané Raich J. (2000) Invertase activity in fresh and processed honeys, J. Sci. Food Agric. 80, 507512.

[SHL 81] Shljakhov P. (1981) Contribution to the investigation of chestnut as bee pasture, XXVIII Int. Beekeep. Congr. Apimondia Acapulco, p. 407.

[STA 74] Stanley R.G., Linskens H.F. (1974) Pollen: biology-biochemistry management. Springer-Verlag, Berlin (quoted by Crane E., Walker P., Day R. (1984) Directory of important world honey sources, IBRA, London).

[THR 95] Thrasyvoulou A., Manikis J. (1995) Some physicochemical and microscopic characteristics of Greek unifloral honeys, Apidologie 26, 441452

[TSI 00] Tsigouri A., Passaloglou Katrali M. (2000) A scientific note on the characteristics of thyme honey from the Greek Island of Kithira, Apidologie $31,457-458$.

[VDo 96] von der Ohe W., von der Ohe K. (1996) Charakterisierung einheimischer Rapshonige, Dtsch. Bienen J. 4, 438-443.

[VOR 64] Vorwohl G. (1964) Die Messung der elektrischen Leitfahigkeit des Honigs und die Verwendung der Messwerte zur Sortendiagnose und Nachweis von Verfalschungen mit Zuckerfutterrungshonigen, Z. Bienenforsch. 7, 37-47.

\section{REFERENCES}

Bogdanov S., Martin P., Lüllmann C. (1997) Harmonised methods of the European Honey Commission, Apidologie extra issue, 1-59.

Persano Oddo L., Piro R. (2004) Main European unifloral honeys: descriptive sheets, Apidologie 35 (Suppl. 1), S38-S81. 